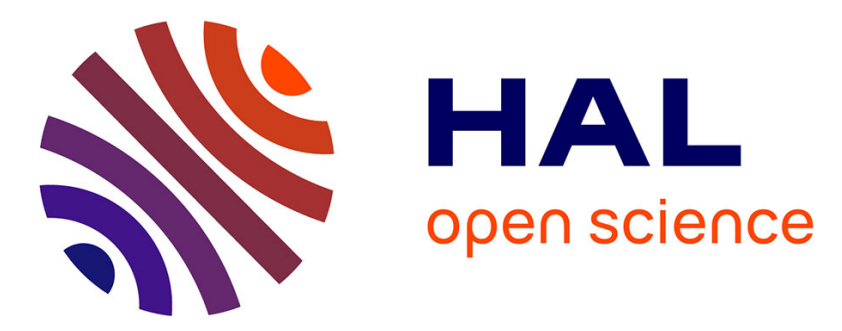

\title{
Effects of nozzle-exit boundary-layer profile on the initial shear-layer instability, flow field and noise of subsonic jets
}

Christophe Bogey, Roberto Sabatini

\section{- To cite this version:}

Christophe Bogey, Roberto Sabatini. Effects of nozzle-exit boundary-layer profile on the initial shearlayer instability, flow field and noise of subsonic jets. Journal of Fluid Mechanics, 2019, 876, pp.288 325. 10.1017/jfm.2019.546. hal-02333791

\section{HAL Id: hal-02333791 \\ https://hal.science/hal-02333791}

Submitted on 24 Nov 2020

HAL is a multi-disciplinary open access archive for the deposit and dissemination of scientific research documents, whether they are published or not. The documents may come from teaching and research institutions in France or abroad, or from public or private research centers.
L'archive ouverte pluridisciplinaire HAL, est destinée au dépôt et à la diffusion de documents scientifiques de niveau recherche, publiés ou non, émanant des établissements d'enseignement et de recherche français ou étrangers, des laboratoires publics ou privés. 


\title{
Effects of nozzle-exit boundary-layer profile on the initial shear-layer instability, flow field and noise of subsonic jets
}

\author{
Christophe Bogey ${ }^{1} \dagger$ and Roberto Sabatini ${ }^{2}$ \\ ${ }^{1}$ Univ Lyon, Ecole Centrale de Lyon, INSA Lyon, Université Claude Bernard Lyon I, CNRS, \\ Laboratoire de Mécanique des Fluides et d'Acoustique, UMR 5509, F-69134, Ecully, France \\ ${ }^{2}$ Departement of Physical Sciences, Embry-Riddle Aeronautical University, \\ Daytona Beach, 32114 Florida, USA \\ (Received $* * *$ )
}

The influence of the nozzle-exit boundary-layer profile on high-subsonic jets is investigated by performing compressible large-eddy simulations (LES) for three isothermal jets at a Mach number of 0.9 and a diameter-based Reynolds number of $5 \times 10^{4}$, and by conducting linear stability analyses from the mean flow fields. At the exit section of a pipe nozzle, the jets exhibit boundary layers of momentum thickness of approximately $2.8 \%$ of the nozzle radius and a peak value of turbulence intensity of $6 \%$. The boundary-layer shape factors, however, vary and are equal to 2.29, 1.96 and 1.71 . The LES flow and sound fields differ significantly between the first jet with a laminar mean exit velocity profile and the two others with transitional profiles. They are close to each other in these two cases, suggesting that similar results would also be obtained for a jet with a turbulent profile. For the two jets with non-laminar profiles, the instability waves in the near-nozzle region emerge at higher frequencies, the mixing layers spread more slowly and contain weaker low-frequency velocity fluctuations, and the noise levels in the acoustic field are lower by $2-3 \mathrm{~dB}$ compared to the laminar case. These trends can be explained by the linear stability analyses. For the laminar boundary-layer profile, the initial shear-layer instability waves are most strongly amplified at a momentum-thickness-based Strouhal number $\mathrm{St}_{\theta}=0.018$, which is very similar to the value obtained downstream in the mixing-layer velocity profiles. For the transitional profiles, on the contrary, they predominantly grow at higher Strouhal numbers, around $\mathrm{St}_{\theta}=0.026$ and 0.032 , respectively. As a consequence, the instability waves rapidly vanish during the boundary-layer/shear-layer transition in the latter cases, but continue to grow over a large distance from the nozzle in the former case, leading to persistent large-scale coherent structures in the mixing layers for the jet with a laminar exit velocity profile.

\section{Introduction}

There has been a considerable amount of studies on the effects of the initial conditions on free shear layers and jets for more than five decades. In particular, a great attention has been paid to the state of the nozzle-exit boundary layer, which may vary from one experiment to another depending on the facility characteristics and on the nozzle diameter and geometry. For instance, the jets are often initially laminar in small-scale experiments, whereas they are initially turbulent in full-scale experiments. In order to make meaningful comparisons, it can therefore be necessary to trip the boundary layer in

$\dagger$ Email address for correspondence: christophe.bogey@ec-lyon.fr 
the nozzle in order to generate turbulent exit conditions, as was the case in the pioneering work of Bradshaw (1966) and Crow \& Champagne (1971).

The differences obtained between initially laminar and initially turbulent shear layers and jets have been described in a long list of papers. In the laminar case, instability waves are amplified just downstream of the nozzle at a preferred momentum-thickness-based Strouhal number equal to $\mathrm{St}_{\theta}=0.017$ according to the linear stability analyses conducted from hyperbolic-tangent velocity profiles (Michalke 1984), and varying within the range $0.009 \leq \mathrm{St}_{\theta} \leq 0.018$ in experiments (Sato 1971; Zaman \& Hussain 1981; Gutmark \& Ho 1983). The shear layers subsequently roll up to form essentially two-dimensional vortical structures, whose interactions result in three-dimensional turbulence. The levels of velocity fluctuations rapidly increase and reach a sharp peak during that laminar-turbulent transition. In the initially turbulent case, on the contrary, they grow monotonically and very slowly from the nozzle exit (Bradshaw 1966; Hill et al. 1976; Browand \& Latigo 1979; Hussain \& Zedan 1978b; Husain \& Hussain 1979). Moreover, the jet flow development is found to be faster in the laminar case than in the turbulent case, leading to a shorter potential core and a higher rate of centerline velocity decay (Hill et al. 1976; Raman et al. 1989, 1994; Russ \& Strykowski 1993; Xu \& Antonia 2002). The impact of the nozzle-exit boundary-layer state is also significant on jet noise sources, as reported in the review papers by Crighton (1981) and Lilley (1994). It has notably been established in Maestrello \& McDaid (1971), Zaman (1985a,b) and Bridges \& Hussain (1987) that initially laminar jets emit more noise than initially turbulent jets, and that the additional acoustic components can be attributed to the pairings of the two-dimensional vortices induced by the laminar-turbulent transition in the shear layers. After the transition, coherent, well-organized turbulent structures appear to persist, as revealed by the experiments of Brown \& Roshko (1974) and Wygnanski et al. (1979). The presence of coherent structures in initially turbulent mixing layers is less obvious according to Chandrsuda et al. (1978), but is supported by the measurements in such flows of a peak Strouhal number of $\mathrm{St}_{\theta}=0.022-0.028$ by Drubka \& Nagib (1981), Hussain \& Zaman (1985) and Morris \& Foss (2003). The reasons for these values of Strouhal number well above those obtained for initially laminar flow conditions remain however unexplained, as was noted by Ho \& Huerre (1984).

The issue of jet initial conditions has recently received renewed attention in the aeroacoustics community since Viswanathan (2004) stated that the jet far-field measurements of Tanna (1977) might be contaminated by spurious facility noise. In reply to this, HarperBourne (2010) suggested that the extra components emerging at high frequencies in Tanna (1977)'s sound spectra are due to laminar flow conditions at the nozzle exit. This seems to be confirmed by the experimental results obtained by Viswanathan \& Clark (2004), Zaman (2012) and Karon \& Ahuja (2013) for high-subsonic jets exhausting from two nozzles of different internal profiles, namely the ASME and the conical nozzles. Indeed, more noise is measured with the ASME nozzle than with the conical nozzle, that is, for highly-disturbed, nominally laminar boundary layers than for turbulent boundary layers, refer to the nozzle-exit conditions of table 1. For instance, for the jet at a Mach number of 0.896 considered by Zaman (2012), the sound levels with the ASME nozzle are stronger by 2-3 $\mathrm{dB}$ for diameter-based Strouhal numbers $\mathrm{St}_{D} \geq 0.3$ at all radiation angles, and approximately by $1 \mathrm{~dB}$ for lower frequencies at angles between 60 and 90 degrees with respect to the flow direction. On the basis of flow visualizations, Zaman (2017) related this to the perseverance of organized coherent structures in the shear layers of the jets issuing from the ASME nozzle. Similarly, in the experiment of Fontaine et al. (2015) who explored the shear-layer flow properties and the noise of three initially highly disturbed jets with different nozzle-exit conditions, given in table 1 , the jet from 


\begin{tabular}{ccccccc}
\hline \multirow{2}{*}{ reference } & case & $\mathrm{Re}_{D}$ & $\mathrm{H}$ & $\delta_{\theta} / r_{0}$ & $\operatorname{Re}_{\theta}$ & $u_{e}^{\prime} / u_{j}$ \\
Zaman (2012) & ASME, $\mathrm{M}=0.37$ & $2.2 \times 10^{5}$ & (laminar) & 0.0050 & 556 & $11.5 \%$ \\
& conical, $\mathrm{M}=0.37$ & $2.2 \times 10^{5}$ & (turbulent) & 0.0106 & 1179 & $7 \%$ \\
Karon \& Ahuja (2013) & ASME, $\mathrm{M}=0.40$ & $3.5 \times 10^{5}$ & 2.34 & 0.0049 & 870 & - \\
& conical, $\mathrm{M}=0.40$ & $3.5 \times 10^{5}$ & 1.71 & 0.0065 & 1135 & - \\
Fontaine et al. (2015) & short nozzle & $6.6 \times 10^{5}$ & 2.18 & 0.0109 & 3620 & $14 \%$ \\
& medium nozzle & $6.6 \times 10^{5}$ & 1.53 & 0.0307 & 10180 & $13 \%$ \\
Brès et al. (2018) & long nozzle & $6.6 \times 10^{5}$ & 1.47 & 0.0426 & 14030 & $12 \%$ \\
& Baseline_LES_10M & $10^{6}$ & 2.54 & 0.0102 & 5100 & $6 \%$ \\
Morris \& Foss (2003) & BL16M_WM_Turb & $10^{6}$ & 1.55 & 0.0142 & 7100 & $13 \%$ \\
& turb. boundary layer & - & 1.31 & - & 4650 & -
\end{tabular}

TABLE 1. Flow conditions at the nozzle exit for round jets (Zaman 2012; Karon \& Ahuja 2013; Fontaine et al. 2015; Brès et al. 2018) and at the separation point created using a sharp edge for a turbulent boundary layer (Morris \& Foss 2003).

the small nozzle with a partially developed boundary layer generates $3 \mathrm{~dB}$ more intense sound than the two jets from the medium and large nozzles with fully turbulent boundary layers. In addition, the peak turbulence intensities a few diameters downstream of the nozzle exit are stronger for the first jet.

The relative importance of each of the nozzle-exit parameters in the above results is difficult to distinguish, because these parameters usually vary simultaneously, as illustrated in table 1 . When the nozzle-exit flow conditions become turbulent, with or without boundary-layer tripping, the shape factor of the boundary-layer profile decreases. This factor, defined as $\mathrm{H}=\delta^{*} / \delta_{\theta}$ where $\delta^{*}$ and $\delta_{\theta}$ are the boundary-layer displacement and momentum thicknesses, takes values around 2.5 for laminar profiles and 1.4 for turbulent profiles. At the same time, the boundary-layer thickness increases, and the nozzle-exit peak turbulence intensities $u_{e}^{\prime} / u_{j}$, where $u_{e}^{\prime}$ and $u_{j}$ are the maximum rms value of velocity fluctuations and the jet velocity, most often grow. In some experiments, similar turbulence levels are obtained, as, for instance, in the work of Morris \& Zaman (2009) where values of $u_{e}^{\prime} / u_{j}$ equal to $6.7 \%$ and $7.5 \%$ are reported for untripped and tripped jets at a diameter-based Reynolds number $\operatorname{Re}_{D}=3 \times 10^{5}$. It even happens that the velocity fluctuations are larger in laminar than in turbulent nozzle-exit boundary layers. Examples of this counter-intuitive tendency have been given by Raman et al. (1989, 1994) for tripped/untripped jets and by Zaman (2012) who measured values of $u_{e}^{\prime} / u_{j}$ around 11\% using the ASME nozzle but around 7\% using the conical nozzle for jets at $2 \times 10^{5} \leq \operatorname{Re}_{D} \leq 6 \times 10^{5}$, see the values for $\operatorname{Re}_{D}=2.2 \times 10^{5}$ in table 1 . In that case, the effects of the velocity profile and those of the turbulence levels are likely to counteract each other, which may result in some confusion.

Therefore, there is clearly a need to study the influence of the nozzle-exit boundarylayer profile with all other exit parameters held constant. For this, it seems worthwhile to use unsteady compressible simulations, which have made spectacular progress over the last three decades, and now allow us to conduct investigations under controlled conditions. Large-eddy simulations (LES) have for instance been run by the first author over the last decade (Bogey \& Bailly 2010; Bogey et al. 2011b,c, 2012a,b; Bogey \& Marsden 2013; Bogey 2018) to investigate the impact of nozzle-exit conditions on initially laminar and highly-disturbed subsonic round jets. Due to limitations in computing resources, the jets had moderate Reynolds numbers $\operatorname{Re}_{D}$ between $2.5 \times 10^{4}$ and $2 \times 10^{5}$, and all 
exhibited laminar mean velocity profiles at the nozzle exit, in order to ensure numerical accuracy. Subsonic jets with tripped boundary layers have also been recently calculated by an increasing number of other researchers, including Lorteau et al. (2015) and Zhu et al. (2018), among others. Specifically concerning initially turbulent jets, the first attempts of computation have been made by Bogey et al. (2008) and Uzun \& Hussaini (2007). However, the grid was too coarse in the former case, while its spatial extent was limited to 4.5 diameters downstream of the nozzle in the latter. Later, Sandberg et al. (2012) carried out the simulation of a fully turbulent pipe flow at $\operatorname{Re}_{D}=7,500$ exiting into a coflow, and Bühler et al. (2014) successfully computed a jet at $\operatorname{Re}_{D}=18,100$ with turbulent conditions at the exit of a pipe nozzle. None of these studies however addresses the question of the mean velocity profile. More recently, two jets at $\operatorname{Re}_{D}=2 \times 10^{5}$ with nozzle-exit conditions roughly matching those found in experiments using the ASME and the conical nozzles have been performed by Bogey \& Marsden (2016). Unfortunately, the results for the two jets are very similar, suggesting that the jet initial conditions in the simulations do not adequately reflect those in the experiments. Finally, Brès et al. (2018) calculated two isothermal subsonic jets at $\operatorname{Re}_{D}=10^{6}$ with initially laminar and turbulent nozzle-exit boundary layers, as indicated in table 1 . The initially laminar jet radiates greater high-frequency noise than the initially turbulent jet, which was attributed to the fact that the instability waves in the near-nozzle region grow at different rates in the two jets.

In the present work, the influence of the nozzle-exit boundary-layer profile on highsubsonic jets is investigated by combining well-resolved large-eddy simulations and linear stability analyses for three isothermal round jets at a Mach number $\mathrm{M}=u_{j} / c_{a}=0.9$ and a Reynolds number $\operatorname{Re}_{D}=u_{j} D / \nu=5 \times 10^{4}$, where $c_{a}, D$ and $\nu$ are the speed of sound in the ambient medium, the jet diameter and the kinematic molecular viscosity. In order to consider the effects of the mean velocity profile alone, momentum boundary-layer thicknesses of $\delta_{\theta} \simeq 0.028 r_{0}$ and peak turbulence intensities of $u_{e}^{\prime} / u_{j} \simeq 6 \%$ are prescribed at the exit of a pipe nozzle for all jets. The boundary-layer profiles however vary, and are laminar in the first jet and transitional (partially developed) in the two others, with shape factors $\mathrm{H}$ ranging between 1.71 and 2.29. The first objective will be to determine whether the flow and sound fields of the jets show significant differences, and whether these differences correspond to those usually encountered between initially laminar and initially turbulent jets, namely a faster flow development, stronger velocity fluctuations in the mixing layers and more noise in the acoustic field in the laminar case. In particular, comparisons will made with the trends observed in the experiments of Zaman (2012, 2017) using the ASME and the conical nozzles and of Fontaine et al. (2015), and in the simulations of Brès et al. (2018). They will be mostly qualitative due to the disparities in upstream flow conditions. The second objective will be to propose an explanation for the higher noise levels expected for a laminar boundary-layer profile. For that purpose, the development of the instability waves very near the nozzle exit and during the transition from a boundary layer to a shear layer will be detailed. It will also be discussed based on the linear stability analyses conducted from the mean flow fields, as in Fontaine et al. (2015) and Brès et al. (2018). However, while the latter authors mainly focused on the amplification rates of the instability waves, the present study will specially examine the sensitivity of the unstable frequencies to the nozzle-exit velocity profile, previously noted by Drubka \& Nagib (1981), Hussain \& Zaman (1985) and Morris \& Foss (2003) for shear layers, and its possible role in the discrepancies observed in the flow and sound fields of the jets.

The paper is organized as follows. The parameters of the three jets, of the largeeddy simulations, of the extrapolations of the LES acoustic near fields to the far field 


$\begin{array}{lcccc} & \mathrm{H} & \delta_{\theta} / r_{0} & \delta_{99} / r_{0} & \alpha_{\text {trip }} \\ \text { jetBL } & 2.55 & 0.0288 & 0.202 & 0.0460 \\ \text { jetT1 } & 1.88 & 0.0288 & 0.215 & 0.0675 \\ \text { jetT2 } & 1.52 & 0.0288 & 0.254 & 0.0830\end{array}$

TABLE 2. Shape factor $H$, momentum thickness $\delta_{\theta}$ and $99 \%$ velocity thickness $\delta_{99}$ of the boundary-layer profile at the pipe-nozzle inlet, and strength of the trip-like excitation $\alpha_{\text {trip }}$.

where $\delta_{B L}$ is the boundary-layer thickness.

In jetT1 and jetT2, the inlet profiles are transitional boundary-layer profiles with $\mathrm{H}=1.88$ and $\mathrm{H}=1.52$, respectively. They are derived from the turbulent profile proposed by De Chant (2005), and defined as

$$
\frac{u_{\text {inlet }}(r)}{u_{j}}=\left\{\begin{array}{l}
\left(\sin \left[\frac{\pi}{2}\left(\frac{r_{0}-r}{\delta_{T_{i}}}\right)^{\beta_{i}}\right]\right)^{\gamma_{i}} \text { if } r \geq r_{0}-\delta_{T_{i}} \\
1 \text { otherwise }
\end{array}\right.
$$


(a)

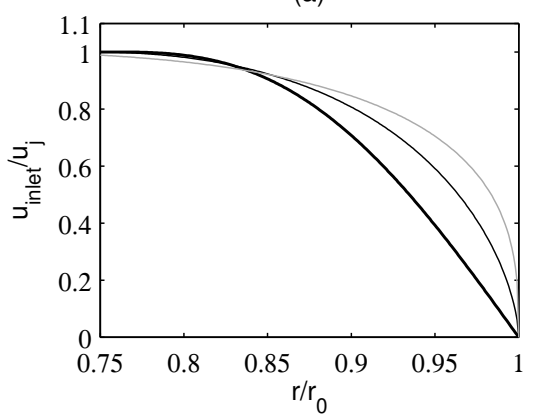

(b)

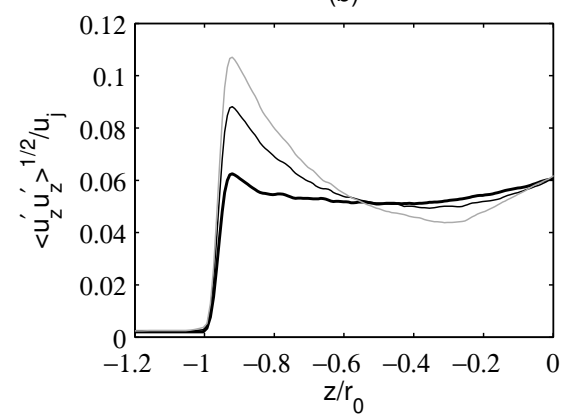

FiguRE 1. Representation (a) of the axial velocity profile $u_{\text {inlet }}$ imposed at the pipe-nozzle inlet and (b) of the peak rms values of axial velocity fluctuations $u_{z}^{\prime}$ in the nozzle: - jetBL, jetT1, - jetT2.

where $\delta_{T_{i}}$ is the boundary-layer thickness, and the values of the exponents $\beta_{i}$ and $\gamma_{i}$ are equal to $\beta_{1}=0.464$ and $\gamma_{1}=1.32$, and to $\beta_{2}=0.423$ and $\gamma_{2}=0.82$. Considering the strong similarities between the near-wall mean-flow statistics obtained for turbulent pipe and boundary layer flows (Monty et al. 2009), they have been designed to fit the experimental data provided by Schubauer \& Klebanoff (1955) for a flat-plate boundary layer in the region of changeover from laminar to fully turbulent conditions, as shown in appendix A.

In the three jets, the inlet boundary-layer thicknesses are arbitrarily set to $\delta_{B L}=0.25 r_{0}$ in jetBL, $\delta_{T_{1}}=1.043 \delta_{B L}=0.26 r_{0}$ in JetT1 and $\delta_{T_{2}}=1.328 \delta_{B L}=0.33 r_{0}$ in JetT2, in order to obtain a momentum thickness of $\delta_{\theta}=0.0288 r_{0}$ in all cases. The associated $99 \%$ velocity thicknesses $\delta_{99}$ thus vary from $0.202 r_{0}$ in jetBL up to $0.254 r_{0}$ in jetT2. With respect to the experiments of Zaman (2012) and Karon \& Ahuja (2013), see in table 1, the boundary layers in the present jets are thicker to guarantee a high numerical accuracy, as will be discussed in section 2.3 and in appendix B. Given the jet Reynolds number of $\operatorname{Re}_{D}=5 \times 10^{4}$, chosen to perform very well resolved LES, this also leads to a momentumthickness-based Reynolds number of $\operatorname{Re}_{\theta}=u_{j} \delta_{\theta} / \nu=720$, which is comparable to the values measured in the experiments. This is of importance because $\operatorname{Re}_{\theta}$ is a key parameter in developing shear layers (Hussain \& Zedan 1978b; Bogey \& Marsden 2013).

In order to generate disturbed upstream conditions for the jets, which otherwise would initially contain negligible velocity fluctuations, the boundary layers are 'tripped' in the pipe using an arbitrary forcing devices whose parameters are determined by trial and error (Klebanoff \& Diehl 1952; Coles 1962; Erm \& Joubert 1991; Schlatter \& Örlü 2012; Hutchings 2012; Castillo \& Johansson 2012). In simulations, forcing devices of different kinds have been proposed. A small step can for instance be mounted on the wall inside the nozzle. Random fluctuations, synthetic turbulence or instability modes can alternatively be imposed on the flow profiles. In the present jets, the forcing procedure detailed in the appendix A of Bogey et al. (2011b) is implemented. It consists in adding random low-level vortical disturbances uncorrelated in the azimuthal direction in the boundary layers. It has been previously used for both laminar (Bogey et al. 2011b, 2012b,a; Bogey \& Marsden 2013) and non-laminar (Bogey \& Marsden 2016) boundary-layer profiles. The forcing is applied at the axial position $z=-0.95 r_{0}$ and at the radial position of $r=r_{0}-\delta_{B L} / 2=0.875 r_{0}$ in all cases. However, the forcing magnitudes are not the same, and have been adjusted after preliminary tests to obtain peak nozzle-exit turbulence intensities $u_{e}^{\prime} / u_{j}$ of $6 \%$ for all jets. This level is close to those measured by Zaman (2012) just downstream of the conical nozzle for initially turbulent jets, refer to 
table 1 for instance. The values of the coefficient $\alpha_{\text {trip }}$ setting the maximum value of the added velocity fluctuations to $\alpha_{\text {trip }} u_{j}$, hence specifying the forcing strength, are given in table 2 . They are equal to $0.046,0.0675$ and 0.083 in jetBL, jetT1 and jetT2, respectively. Consequently, the lower the inlet boundary-layer shape factor, the higher the amplitude of the excitation necessary to reach $u_{e}^{\prime} / u_{j}=6 \%$, This is illustrated in figure 1(b) showing the variations of the maximum rms value of axial velocity fluctuations in the pipe.

As pointed out above, there exit some discrepancies between the nozzle-exit conditions of the present jets and of the experiments of table 1 in terms of $\operatorname{Re}_{D}$ and ratio $\delta_{\theta} / r_{0}$. The higher value of $\delta_{\theta} / r_{0}$ in the simulations, in particular, will result in lower frequencies in the shear layers just downstream of the nozzle. Thanks to the similarities in $\operatorname{Re}_{\theta}$ and $u_{e}^{\prime} / u_{j}$, the physical mechanisms at play in this zone can yet be expected to be of the same nature as those in the experiments using the ASME and conical nozzles. Performing qualitative comparisons with the trends revealed in these experiments therefore appears relevant. Quantitative comparisons with measurements for reference jets of the literature will also be made throughout the paper. They are given mainly for illustration purposes, because these jets have Reynolds numbers $\operatorname{Re}_{D} \simeq 10^{6}$ and certainly very thin nozzle-exit boundary layers. In addition, they are most likely initially fully turbulent, and such a case is not considered in this study. It is however hoped that on the basis of the differences obtained between jetT1 and jetT2, results for a more turbulent boundary-layer profile could be extrapolated. Finally, the experimental jets all exhaust for a convergent nozzle, leading to a pressure gradient at the nozzle exit whose effects are unclear (Zaman 2012), which is not taken into account in the simulations.

\subsection{LES numerical methods}

For the LES, the numerical framework is identical to that used in previous jet simulations (Bogey \& Bailly 2010; Bogey et al. 2012b, 2011b, 2012a; Bogey \& Marsden 2013; Bogey 2018). They are carried out using an in-house solver of the three-dimensional filtered compressible Navier-Stokes equations in cylindrical coordinates $(r, \theta, z)$ based on low-dissipation and low-dispersion explicit schemes. The axis singularity is taken into account by the method of Mohseni \& Colonius (2000). In order to alleviate the time-step restriction near the cylindrical origin, the derivatives in the azimuthal direction around the axis are calculated at coarser resolutions than permitted by the grid (Bogey et al. 2011a). For the points closest to the jet axis, they are evaluated using 16 points, yielding an effective resolution of $2 \pi / 16$. Fourth-order eleven-point centered finite differences are used for spatial discretization, and a second-order six-stage Runge-Kutta algorithm is implemented for time integration (Bogey \& Bailly 2004). A sixth-order eleven-point centered filter (Bogey et al. 2009b) is applied explicitly to the flow variables every time step. Non-centered finite differences and filters are also used near the pipe walls and the grid boundaries (Berland et al. 2007; Bogey \& Bailly 2010). At the boundaries, the radiation conditions of Tam \& Dong (1996) are applied, with the addition at the outflow of a sponge zone combining grid stretching and Laplacian filtering (Bogey \& Bailly 2002). At the inflow and radial boundaries, density and pressure are also brought back close to $p_{a}$ and $\rho_{a}$ every $0.055 r_{0} / c_{a}$ at rate of $0.5 \%$, in order to keep the mean values of density and pressure around their ambient values without generating significant acoustic reflections. No co-flow is imposed.

In the present large-eddy simulations, the explicit filtering is employed to remove gridto-grid oscillations, but also as a subgrid-scale high-order dissipation model in order to relax turbulent energy from scales at wave numbers close to the grid cut-off wave number while leaving larger scales mostly unaffected. The performance of this LES approach has been assessed in past studies for subsonic jets, Taylor-Green vortices and turbulent chan- 


$$
\begin{array}{ccccccc}
n_{r} \times n_{\theta} \times n_{z} & \Delta r / r_{0} & \left(r_{0} \Delta \theta\right) / r_{0} & \Delta z / r_{0} & L_{r} / r_{0} & L_{z} / r_{0} & T u_{j} / r_{0} \\
504 \times 1024 \times 2085 & 0.36 \% & 0.61 \% & 0.72 \% & 15 & 40 & 500
\end{array}
$$

TABLE 3. Numbers of grid points $n_{r}, n_{\theta}$ and $n_{z}$, mesh spacings $\Delta r$ at $r=r_{0}, r_{0} \Delta \theta$ and $\Delta z$ at $z=0$, extents of the physical domain $L_{r}$ and $L_{z}$, and simulation time $T$ after the transient period.

nel flows (Bogey et al. 2011b; Bogey \& Bailly 2006, 2009; Fauconnier et al. 2013; Kremer \& Bogey 2015), from comparisons with the solutions of direct numerical simulations and from the examination of the magnitude and the properties of the filtering dissipation in the wavenumber space.

\subsection{Simulation parameters}

The grid used in the present jet simulations is detailed and referred to as gridz40B in a recent grid-sensitivity study of the flow and acoustic fields of an initially highly disturbed isothermal round jet at $\mathrm{M}=0.9$ and $\operatorname{Re}_{D}=10^{5}$ (Bogey 2018). As indicated in table 3, it contains $n_{r} \times n_{\theta} \times n_{z}=504 \times 1024 \times 2048=10^{9}$ points. It extends radially out to $L_{r}=15 r_{0}$ and axially, excluding the 100-point outflow sponge zone, down to $L_{z}=40 r_{0}$. There are 169 points along the pipe nozzle between $z=-2 r_{0}$ and $z=0,96$ points between $r=0$ and $r=r_{0}$, and 41 points between $r=r_{0}-\delta_{B L}=0.75 r_{0}$ and $r=r_{0}$. In the radial direction, the mesh spacing $\Delta r$ is minimum and equal to $0.0036 r_{0}$ at $r=r_{0}$, and is equal to $0.0141 r_{0}$ at $r=0,0.0148 r_{0}$ at $r=2 r_{0}, 0.0335 r_{0}$ at $r=4 r_{0}$ and $0.075 r_{0}$ between $r=6.25 r_{0}$ and $r=L_{r}$. The latter mesh spacing leads to a diameter-based Strouhal number of $\mathrm{St}_{D}=f D / u_{j}=5.9$ for an acoustic wave discretized by five points per wavelength, where $f$ is the frequency. In the axial direction, the mesh spacing $\Delta z$ is minimum and equal to $0.0072 r_{0}$ between $z=-r_{0}$ and $z=0$, and increases at a stretching rate of $0.103 \%$ farther downstream to reach $0.0127 r_{0}$ at $z=5 r_{0}, 0.0178 r_{0}$ at $z=10 r_{0}$, $0.0230 r_{0}$ at $z=15 r_{0}$ and $0.0488 r_{0}$ at $z=L_{z}$.

The quality of gridz40B has been shown in Bogey (2018) for a jet at $\operatorname{Re}_{D}=10^{5}$ characterized, at the nozzle exit, by a laminar Blasius boundary layer of thickness $\delta_{B L}=$ $0.25 r_{0}$ and a peak turbulence intensity of $u_{e}^{\prime} / u_{j}=9 \%$. Therefore, it is highly likely that in the present work, the grid resolution is appropriate for jetBL at $\operatorname{Re}_{D}=5 \times 10^{4}$ with $\delta_{B L}=0.25 r_{0}$ and $u_{e}^{\prime} / u_{j}=6 \%$. For jetT1 and jetT2 with non-laminar boundary-layer profiles, the suitability of the grid is less obvious. In order to address this issue, the nearwall mesh spacings in the pipe expressed in wall units based on the wall friction velocity at the nozzle exit are provided in table 4 . They are such that $\Delta r^{+} \leq 2.7,\left(r_{0} \Delta \theta\right)^{+} \leq 4.6$ and $\Delta z^{+} \leq 5.4$. The azimuthal and axial mesh spacings meet the requirements needed to compute turbulent wall-bounded flows accurately using direct numerical simulation (Kim et al. 1987; Spalart 1988) or LES involving relaxation filtering (Gloerfelt \& Berland 2012; Kremer \& Bogey 2015). On the contrary, the wall-normal spacing is two or three times larger than the recommended value of $\Delta r^{+}=1$. For the simulation of an initially fully turbulent jet, refining the wall-normal region by a factor of at least three would therefore be necessary, which would increase by the same amount the computational cost due to the explicit time-integration scheme. For the initially transitional jets considered in this paper, the sensitivity to the wall-normal spacing has however been assessed in a preliminary study using two shorter grids extending axially, excluding the outflow sponge zones, only down to $z=4 r_{0}$ in order to save computational time. The coarsest of the two grids coincides with gridz40B in the boundary-layer region. The finest one is also identical to the latter in that region in the directions $\theta$ and $z$, but differs in the radial direction 


$\begin{array}{cccc} & \Delta r^{+} & \left(r_{0} \Delta \theta\right)^{+} & \Delta z^{+} \\ \text {JetBL } & 1.4 & 2.4 & 2.8 \\ \text { JetT1 } & 2.1 & 3.6 & 4.3 \\ \text { JetT2 } & 2.7 & 4.6 & 5.4\end{array}$

TABLE 4. Near-wall mesh spacings $\Delta r, r_{0} \Delta \theta$ and $\Delta z$ given in wall units based on the wall friction velocity $u_{\tau}$ at the nozzle exit.

with $\Delta r / r_{0}=0.18 \%$, instead of $\Delta r / r_{0}=0.36 \%$, at $r=r_{0}$. The tripping procedure is exactly the same in all cases, but the time step is twice as small in the LES using the finest grid because of the numerical stability condition, leading to an application of the relaxation filtering that is twice as frequent. The mean and fluctuating velocity profiles obtained at the nozzle exit using the two grids, represented in Appendix B for jetT2, are superimposed. This demonstrates that the LES solutions in the pipe do not depend on the radial mesh spacing at $r=r_{0}$ or on the relaxation filtering.

In the three jet LES, the time step is defined by $\Delta t=0.7 \times \Delta r\left(r=r_{0}\right) / c_{a}$, yielding $\Delta t=0.0023 \times r_{0} / u_{j}$. After a transient period of $275 r_{0} / u_{j}$, the simulation time $T$, given in table 3 , is equal to $500 r_{0} / u_{j}$. During that time period, the signals of density, velocities and pressure obtained on the jet axis at $r=0$, on the cylindrical surfaces located at $r=r_{0}$ and $r=L_{r}=15 r_{0}$ and in the cross sections at $z=-1.5 r_{0}, z=0$ and $z=L_{z}=40 r_{0}$, are recorded at a sampling frequency allowing spectra to be computed up to $\mathrm{St}_{D}=12.8$. The signals obtained in the four azimuthal planes at $\theta=0, \pi / 4, \pi / 2$ and $3 \pi / 4$ are also stored, but at a halved frequency in order to reduce storage requirements. Finally, the Fourier coefficients estimated over the full section $(r, z)$ for the first nine azimuthal modes for density, velocities and pressure are similarly saved. The flow and acoustic near field statistics presented in the next sections are calculated from these recordings. They are averaged in the azimuthal direction, when possible. Time spectra are evaluated from overlapping samples of duration $45 r_{0} / u_{j}$ on the jet axis, and $90 r_{0} / u_{j}$ otherwise. In the azimuthal direction, post-processing can be performed up to the mode $n_{\theta}=128$, where $n_{\theta}$ is the dimensionless azimuthal wave number such that $n_{\theta}=k_{\theta} r$.

Finally, the simulations required $200 \mathrm{~GB}$ of memory and have run during 340,000 iterations each. They have been performed using an OpenMP-based in-house solver on single nodes with 256 GB of memory, consisting of four Intel Sandy Bridge E5-4650 8core processors at a clock speed of $2.7 \mathrm{GHz}$ or of two Intel Xeon CPU E5-2670v3 8-core processors at $2.6 \mathrm{GHz}$. The time per iteration is approximately equal to 120 seconds in the first case using 32 cores and to 140 seconds in the second case using 16 cores, leading to the consumption of 1,070 and $620 \mathrm{CPU}$ hours, respectively, for 1,000 iterations. Therefore, a total number of the order of 1 billion computational hours has been necessary for the full study.

\subsection{Linear stability analysis}

Inviscid spatial stability analyses have been carried out from the mean flow fields of the jets, as was done in previous investigations (Fontaine et al. 2015; Brès et al. 2018). More precisely, the compressible Rayleigh equation (Michalke 1984; Sabatini \& Bailly 2015) has been solved for the LES profiles of mean axial velocity and mean density, locally considered parallel, from $z=0.02 r_{0}$ down to $z=5 r_{0}$. Viscous effects are not taken into account because they are expected to be very weak at the Reynolds numbers $\operatorname{Re}_{\theta} \gtrsim 700$ considered in this work (Morris 1976, 2010). For a given axial distance $z$ and a given Strouhal number $\mathrm{St}_{D}$, the compressible Rayleigh equation is solved through a shooting 
technique (Morris 2010), based on the Euler method for the integration step and on the secant method for the search of the complex wavenumber $k_{z} \delta_{\theta}$. The integration is performed on a grid with a spatial step of $0.0001 r_{0}$, extending from the LES grid point closest to the jet axis at $r=0.007 r_{0}$ out to $r=5 r_{0}$. Since the present stability study is performed directly from the LES profiles, which may contain high-frequency noise in the near-nozzle region of high mean-flow gradients, the profiles and their radial derivatives are filtered using a sixth-order eleven-point centered filter (Bogey et al. 2009b). A cubic spline interpolation is then employed to calculate the mean-flow values on the aforesaid grid. It can be noted that, in order to check the sensitivity of the results to the filtering, a tenth-order eleven-point centered filter has also been used to smooth the LES profiles of jetT2, in the case which exhibits the strongest gradients. The eigenvalues $k_{z} \delta_{\theta}$ thus obtained are identical to those calculated using the sixth-order filter.

\subsection{Far-field extrapolation}

The LES near-field fluctuations have been propagated to the far field using an in-house OpenMP-based solver of the isentropic linearized Euler equations (ILEE) in cylindrical coordinates, based on the same numerical methods as the LES (Bogey et al. 2009a; Bogey 2018). The extrapolations are carried out from the velocity and pressure fluctuations recorded on the cylindric surface at $r=L_{r}=15 r_{0}$ and on the axial sections at $z=-1.5 r_{0}$ and $z=L_{z}=40 r_{0}$ over a time period of $500 r_{0} / u_{j}$ during the jet simulations, at a sampling frequency corresponding to $\mathrm{St}_{D}=12.8$. They aim to provide the pressure waves radiated at a distance of $150 r_{0}$ from the nozzle exit, where far-field acoustic conditions are expected to apply according to measurements (Ahuja et al. 1987; Viswanathan 2006), between the angles of $\phi=15^{\circ}$ and $\phi=165^{\circ}$ relative to the jet direction.

In practice, in order to compute separately the downstream and the upstream acoustic fields, whose magnitudes strongly vary, two far-field extrapolations are performed on two different grids, yielding results for $15^{\circ} \leq \phi \leq 90^{\circ}$ and for $60^{\circ} \leq \phi \leq 165^{\circ}$, respectively. The two grids are identical in the radial and the azimuthal directions, with $n_{r}=2058$ and $n_{\theta}=256$. In the direction $r$, they extend from $r=2.5 r_{0}$ out to $r=151 r_{0}$ with a mesh spacing of $\Delta r=0.075 r_{0}$, and end with a 80-point sponge zone. In the axial direction, the two grids respectively contain $n_{z}=2171$ and $n_{z}=3111$ points, and extend, excluding the 80-point sponge zones implemented at the upstream and downstream boundaries, from $z=-6 r_{0}$ up to $z=146 r_{0}$ and from $z=-146 r_{0}$ up to $z=76 r_{0}$, with a mesh spacing of $\Delta z=0.075 r_{0}$. This mesh spacing, leading to a Strouhal number $\mathrm{St}_{D}=5.9$ for an acoustic wave discretized by five points per wavelength, is identical to that in the LES near field.

In the first computation, the LES fluctuations are imposed onto the extrapolation grid for $-1.5 r_{0} \leq z \leq L_{z}$ at $r=L_{r}=15 r_{0}$, for $2.5 r_{0} \leq r \leq L_{r}$ at $z=-1.5 r_{0}$ and for $7.5 r_{0} \leq$ $r \leq L_{r}$ at $z=L_{z}=40 r_{0}$. The opening angle relative to the flow direction, with the nozzle exit as origin, is of $\phi=10^{\circ}$, which allows most of the downstream noise components to be taken into account. In the second computation, the LES data are imposed onto the extrapolation grid as in the first one at $z=-1.5 r_{0}$ and at $r=L_{r}=15 r_{0}$, but only for $14 r_{0} \leq r \leq L_{r}$ at $z=L_{z}=40 r_{0}$. The opening angle is larger than in the first case in order to avoid the presence of aerodynamic disturbances (Arndt et al. 1997) on the extrapolation surface, which might cause low-frequency spurious waves (Bogey \& Bailly 2010) in the upstream direction where noise levels are much lower than in the downstream direction.

Each ILEE computation requires 105 or 150 GB of memory depending on the grid used, and lasts during 7,700 iterations. This leads to a total number approximately of 25,000 CPU hours consumed using 16-core nodes based on Intel Xeon CPU E5-2670 processors 
at $2.6 \mathrm{GHz}$. Finally, the far-field spectra are evaluated from the pressure signals obtained at $150 r_{0}$ from the nozzle exit during the final 6,000 iterations of the computations, i.e. during nearly $470 r_{0} / u_{j}$. Thus, for the peak Strouhal number of $\mathrm{St}_{D}=0.2$ emerging in the downstream direction, and for the lowest Strouhal number of $\mathrm{St}_{D}=0.075$ represented in section 3.4.2, the far-field signals contains 48 and 18 time periods, respectively. The statistical convergence of the results is furthermore increased by calculating the spectra using overlapping samples of duration $90 r_{0} / u_{j}$, and by averaging in the azimuthal direction.

\section{Results}

\subsection{Jet flow initial conditions}

\subsubsection{Nozzle-exit boundary-layer properties}

The profiles of mean and rms axial velocities calculated at the nozzle exit are presented in figure 2. Their main properties are provided in table 5. In figure 2(a), as intended, the mean velocity profiles differ significantly, and have shape factors $\mathrm{H}$ of 2.29 for jetBL, 1.96 for jetT1 and 1.71 for jetT2. The boundary-layer momentum thicknesses are similar, and range only from $\delta_{\theta}=0.0299 r_{0}$ for jetBL down to $\delta_{\theta}=0.0274 r_{0}$ for jetT2, leading to Reynolds numbers $\operatorname{Re}_{\theta}$ between 685 and 747 . From jetBL to jetT2, in addition, the $99 \%$ velocity thickness $\delta_{99}$ increases slightly and the vorticity thickness $\delta_{\omega}=\left\langle u_{z}\right\rangle(r=0) / \max \left(\left|\partial\left\langle u_{z}\right\rangle / \partial r\right|\right)$ evaluated from the maximum value of velocity gradient strongly decreases from $\delta_{\omega}=0.118 r_{0}$ down to $\delta_{\omega}=0.043 r_{0}$. The mean velocity profile for jetBL corresponds to a laminar boundary-layer profile, and, given that $\mathrm{H} \simeq 1.45$ is obtained (Spalart 1988; Erm \& Joubert 1991; Fernholz \& Finley 1996; Schlatter \& Örlü 2012) for fully developed boundary layers at $\operatorname{Re}_{\theta}=700$, the profiles for jetT1 and jetT2 are both transitional.

In figure 2(b), the peak turbulence intensities, imposed by the boundary-layer forcing, are all close to $u_{e}^{\prime} / u_{j}=6.1 \%$. They are reached roughly at the positions of the maximum velocity gradients, hence move nearer to the wall from $r_{e}=0.935 r_{0}$ for jetBL up to $r_{e}=$ $0.975 r_{0}$ for jetT2, as reported in table 5 . The radial profile of rms velocity also changes with the boundary-layer shape. In the non-laminar cases, compared to the laminar case (Zaman 1985 $a, b$ ), the peak is sharper and resembles that obtained in the inner region of turbulent boundary layers (Spalart 1988; Schlatter \& Örlü 2012) as well as that measured just downstream of the nozzle lip for such flows (Morris \& Foss 2003; Fontaine et al. 2015).

With respect to the parameters of the inlet boundary layers in table 2, the nozzleexit parameters in table 5 are slightly different due to the flow development in the pipe between the forcing at $z=-0.95 r_{0}$ and the exit at $z=0$. The boundary layer has a lower shape factor and a larger momentum thickness at the exit than at the pipe inlet for jetBL, whereas the opposite trends are observed for the two other jets.

The profiles of the skewness and kurtosis factors of the axial velocity fluctuations at $z=0$ are depicted in figure 3. As expected, significant deviations from the values of 0 and 3 are found in the interfaces between the laminar inner-pipe region and the highly disturbed boundary layers, around $r=0.75 r_{0}$. They are stronger, in absolute value, as the mean velocity profile has a more turbulent shape, and indicate the occurrence of intermittent bursts of low-velocity fluid. In the boundary layers, the strongest deviations are obtained for the laminar case, close to the wall as well as on the high-speed side of the boundary layers. For instance, at $r=r_{0}-\delta_{94}=0.827 r_{0}$ where $\delta_{94}$ is the $94 \%$ velocity boundary-layer thickness, equal to $0.173 r_{0}$ for all jets, the skewness values are of -0.65 for JetBL, -0.43 for JetT1 and -0.28 for JetT2. This tendency is in agreement with that 
(a)

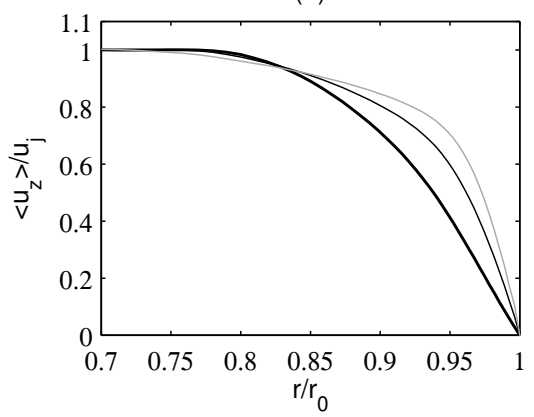

(b)

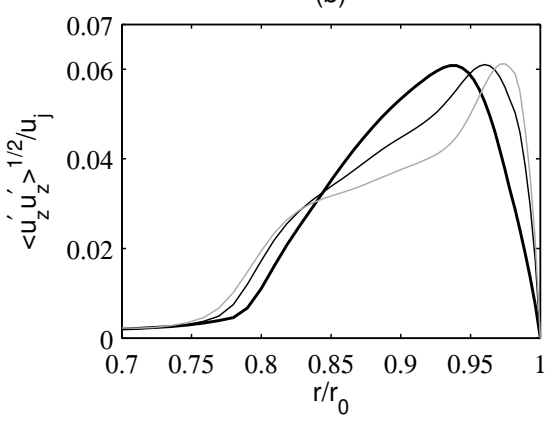

Figure 2. Radial profiles at the nozzle exit at $z=0$ (a) of mean axial velocity $\left\langle u_{z}\right\rangle$ and (b) of the rms values of axial velocity fluctuations $u_{z}^{\prime}$ :

jetBL,

jetT1,

jetT2.

$\mathrm{H} \quad \delta_{\theta} / r_{0} \quad \delta_{99} / r_{0} \quad \delta_{\omega} / r_{0} \quad \operatorname{Re}_{\theta} \quad u_{e}^{\prime} / u_{j} r_{e} / r_{0} n_{\theta}$

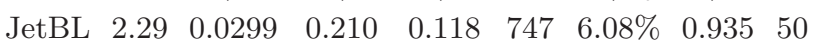

$\begin{array}{lllllllll}\text { JetT1 } & 1.96 & 0.0280 & 0.220 & 0.062 & 700 & 6.10 \% & 0.960 & 51\end{array}$

$\begin{array}{lllllllll}\text { JetT2 } & 1.71 & 0.0274 & 0.241 & 0.043 & 685 & 6.12 \% & 0.975 & 64\end{array}$

TABLE 5. Nozzle-exit parameters: shape factor H, momentum thickness $\delta_{\theta}, 99 \%$ velocity thickness $\delta_{99}$ and vorticity thickness $\delta_{\omega}$ of the boundary-layer profile, Reynolds number $\operatorname{Re}_{\theta}=u_{j} \delta_{\theta} / \nu$, value $u_{e}^{\prime} / u_{j}$ and radial position $r_{e}$ of peak axial turbulence intensity, and peak azimuthal mode $n_{\theta}$ at $r=r_{e}$.

(a)

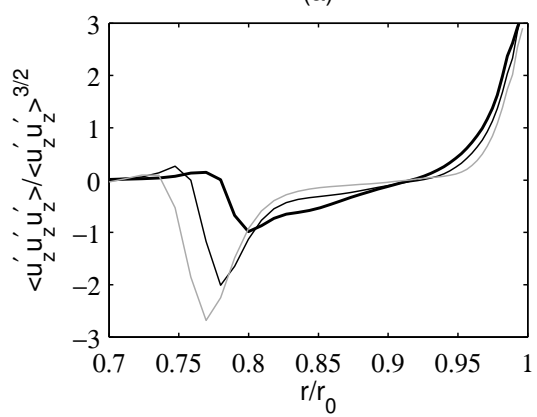

(b)

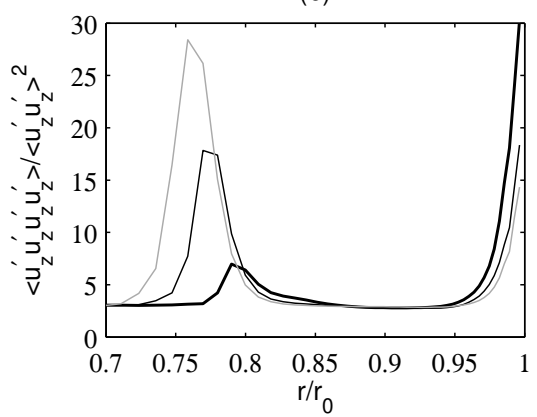

Figure 3. Radial profiles at the nozzle exit (a) of the skewness factor and (b) of the kurtosis factor of axial velocity fluctuations $u_{z}^{\prime}:-\operatorname{jetBL}, \stackrel{-}{-}{ }^{-}$jetT1,

obtained by Zaman (2017) who measured, also at $r=r_{0}-\delta_{94}$, lower values of velocity skewness for nominally laminar nozzle-exit conditions than for turbulent ones.

The properties of the jet initial disturbances are examined by computing spectra of axial velocity fluctuations at the nozzle exit in both the inner and the outer boundarylayer regions. The spectra estimated in the inner region at the position $r=r_{e}$ of the turbulence intensity peak, i.e. between $r_{e}=0.935 r_{0}$ for jetBL and $r_{e}=0.975 r_{0}$ for jetT2, are represented as a function of the Strouhal number $\mathrm{St}_{D}$ in figure $4(\mathrm{a})$ and of the azimuthal mode $n_{\theta}$ in figure $4(\mathrm{~b})$. Their shapes are roughly the same in the three cases, and correspond, as was discussed in a specific note (Bogey et al. 2011c), to the spectral shapes encountered for turbulent wall-bounded flows because of the presence of large-scale elongated structures. As the boundary-layer profile changes from laminar 
(a)

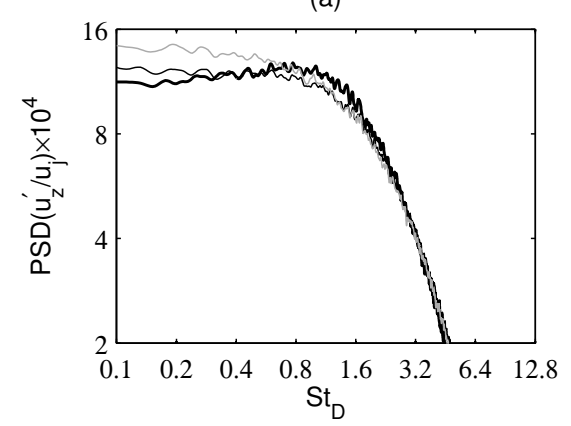

(b)

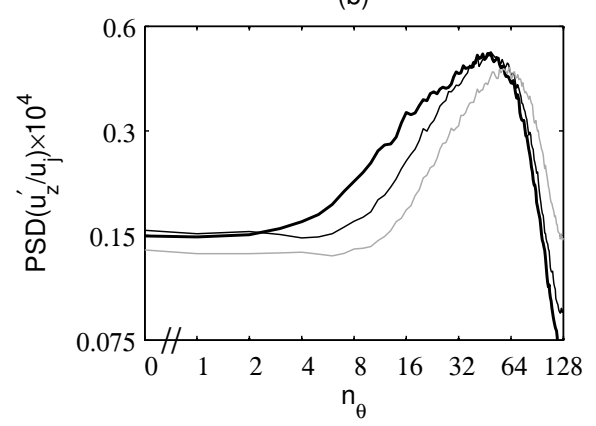

Figure 4. Power spectral densities (PSD) of axial velocity fluctuations $u_{z}^{\prime}$ obtained at the nozzle exit at the position $r=r_{e}$ of peak axial turbulence intensity, as a function (a) of Strouhal number $\mathrm{St}_{D}$ and (b) of azimuthal mode $n_{\theta}:-$ jetBL, $\longrightarrow$ jetT1, $\longrightarrow$ jetT2.

to turbulent, the magnitude of the low-frequency components at $\mathrm{St}_{D}<0.8$ slightly strengthens in figure 4(a), which may be linked to the larger $99 \%$ velocity thickness of the profile. Most obviously, the dominant components in figure 4(b) shift towards higher modes, resulting in peaks at $n_{\theta}=50$ for jetBL, $n_{\theta}=51$ for jetT1 and $n_{\theta}=64$ for jetT2, as reported in table 5 . The turbulent structures are thus spaced out by $\lambda_{\theta}=0.13 r_{0}$, $\lambda_{\theta}=0.12 r_{0}$ and $\lambda_{\theta}=0.10 r_{0}$, respectively. The modification of their spatial arrangement in the azimuthal direction may be related to the increase of the velocity gradient.

The spectra evaluated in the outer boundary-layer region at $r=r_{0}-\delta_{94}=0.827 r_{0}$ in all cases are depicted in figures 5 . Their levels are normalized by the rms values of velocity fluctuations at this position, equal to $\left\langle u_{z}^{\prime 2}\right\rangle^{1 / 2}=0.0248 u_{j}$ for JetBL, $0.0283 u_{j}$ for JetT1 and $0.0285 u_{j}$ for JetT2. The spectra are very similar to each other, both in shape and in amplitude. Compared to the near-wall spectra, two important differences can be noticed. First, a significant amount of energy is contained by the components centered around a Strouhal number of $\mathrm{St}_{D}=3.2$ in figure $5(\mathrm{a})$, whereas a rapid collapse is observed for $\mathrm{St}_{D} \geq 1.6$ in figure $4(\mathrm{a})$. Second, the dominant mode in the azimuthal direction is $n_{\theta} \simeq 40$ for all cases in figure $5(\mathrm{~b})$, whereas it is higher, and increases for a lower boundary-layer shape factor in figure 4(b). Therefore, the turbulent structures organize differently near the wall and further away, as expected (Tomkins \& Adrian 2005). Furthermore, they appear to depend on the form of the velocity profile in the first region, but not in the second one.

\subsubsection{Very near-nozzle instability waves}

In order to characterize the instability waves initially growing in the shear layers, an inviscid linear stability analysis is carried out following the methodology described in section 2.4 from the LES mean flow profiles at $z=0.1 r_{0}$, corresponding to $z \simeq 3.6 \delta_{\theta}(0)$ in terms of nozzle-exit boundary-layer momentum thickness $\delta_{\theta}(0)$. The mean velocity profiles at this position are shown in figure 6(a). They are very similar to the nozzle-exit profiles of figure 2(a), and have momentum thicknesses only $2 \%$ larger than the exit values reported in table 5 . This persistence of the mean velocity profile is in agreement with the measurements of Morris \& Foss (2003) downstream of a sharp corner for a turbulent boundary layer at $\operatorname{Re}_{\theta}=4650$, as indicated in table 1 . For the comparison, a hyperbolictangent velocity profile with $\delta_{\theta}=0.0288 r_{0}$, that is the momentum thickness imposed at the pipe-nozzle inlet, is also plotted. This type of analytical profile is often used in linear stability analyses for mixing layers and jets (Michalke 1984), providing good predictions of the peak Strouhal number $\mathrm{St}_{\theta}$ for initially laminar conditions (Gutmark \& Ho 1983), 
(a)

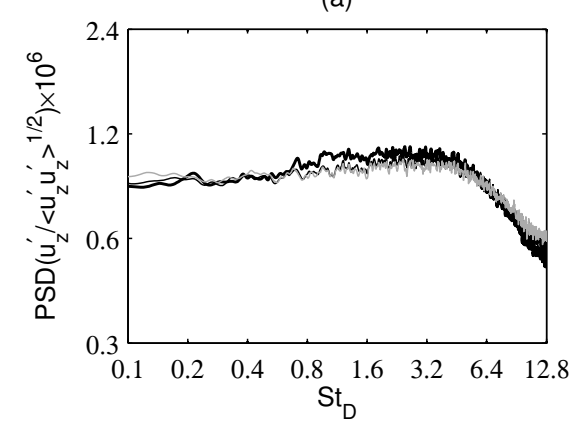

(b)

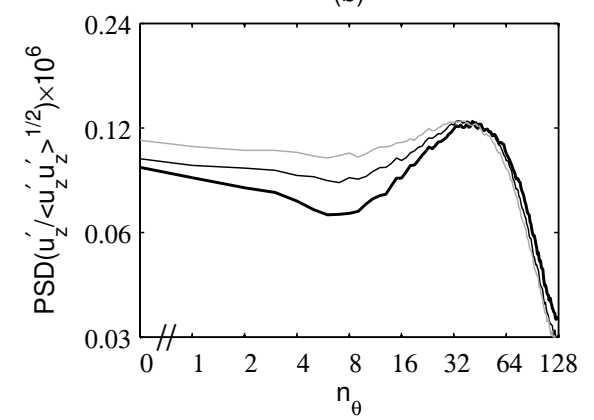

Figure 5. Power spectral densities of axial velocity fluctuations $u_{z}^{\prime}$ obtained at the nozzle exit at $r=r_{0}-\delta_{94}$, as a function (a) of Strouhal number $\mathrm{St}_{D}$ and (b) of azimuthal mode $n_{\theta}$ : jetBL, $\longrightarrow$ jetT1, $\longrightarrow$ jetT2.

but poor ones for initially turbulent conditions (Drubka \& Nagib 1981; Hussain \& Zaman 1985).

The instability amplification rates $-\operatorname{Im}\left(k_{z}\right) \delta_{\theta}$ computed for the first two azimuthal modes $n_{\theta}=0$ and $n_{\theta}=1$ are represented in figure $6(\mathrm{~b})$ as a function of the Strouhal number $\mathrm{St}_{\theta}$. Their peak frequencies are gathered in table 6 . The curves obtained for the two modes are superimposed, due to the value of $\delta_{\theta} / r_{0}<1 / 25$ (Michalke 1984), with a slight predominance of the axisymmetric mode. Their sensitivity to the velocity profile is much more spectacular. For jetBL, the range of unstable frequencies is narrower and the peak growth rate is higher than those for the hyperbolic-tangent profile. Despite these discrepancies, the peak grow rates are reached at very similar Strouhal numbers, namely $\mathrm{St}_{\theta}=0.018$ for jetBL and $\mathrm{St}_{\theta}=0.017$ for the analytical profile. For the two other jets, the range of unstable frequencies broaden and the growth rates strengthen as the exit profile deviates from a laminar profile. In addition, the peak Strouhal number increases to $\mathrm{St}_{\theta}=0.026$ for jetT1 and to $\mathrm{St}_{\theta}=0.032$ for jetT2.

The present changes in peak frequency at $z=0.1 r_{0} \simeq 3.6 \delta_{\theta}(0)$ depending on the boundary-layer profile are consistent with the data of the literature. For instance, the peak Strouhal numbers of $\mathrm{St}_{\theta}=0.022-0.028$ measured by Drubka \& Nagib (1981) and Hussain \& Zaman (1985) in initially turbulent mixing layers are greater than those found around $\mathrm{St}_{\theta}=0.013$ in initially laminar mixing layers. Closer to this study, in the experiments of Morris \& Foss (2003), a hump emerges at $\mathrm{St}_{\theta} \simeq 0.06$ in the velocity spectrum acquired $3.54 \delta_{\theta}(0)$ downstream of a sharp edge, where $\delta_{\theta}(0)$ here denotes the boundary-layer momentum thickness at the edge. Finally, the linear stability analyses performed at $z=0.08 r_{0}$ in Fontaine et al. (2015) and at $z=0.16 r_{0}$ in Brès et al. (2018) for the jets reported in table 1 also reveal peak amplification rates at higher $\mathrm{St}_{\theta}$ for turbulent than for laminar nozzle-exit flow conditions. Indeed, while the peak Strouhal numbers emerge at $\mathrm{St}_{\theta}=0.012-0.014$ for the short-nozzle case in Fontaine et al. (2015) and for Baseline_LES_10M in Brès et al. (2018), they are equal to $\mathrm{St}_{\theta}=0.09$ for the long-nozzle case and to $\mathrm{St}_{\theta}=0.024$ for BL16M_WM_Turb. Remark that the positions of $z=0.08 r_{0}$ for the long-nozzle case and of $z=0.16 r_{0}$ for BL16M_WM_Turb correspond respectively to $z=1.9 \delta_{\theta}(0)$ and to $z=11 \delta_{\theta}(0)$. The variations of the peak frequencies with the axial position will be discussed later in section 3.2.3.

Instead of the momentum thickness, the peak frequency of the instability growth rates can be related to other length scales of the velocity profiles, such as the vorticity thickness $\delta_{\omega}$ or viscous wall units at the nozzle exit, as proposed by Morris \& Foss (2003). 
(a)

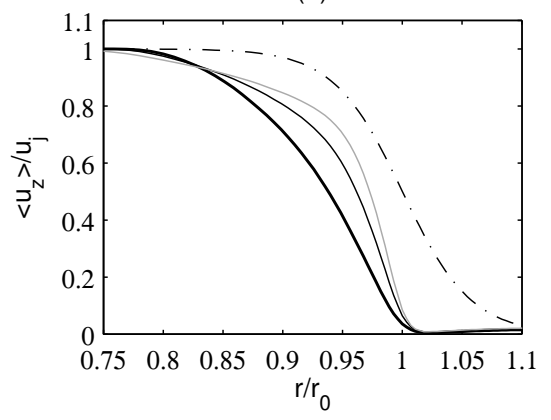

(b)

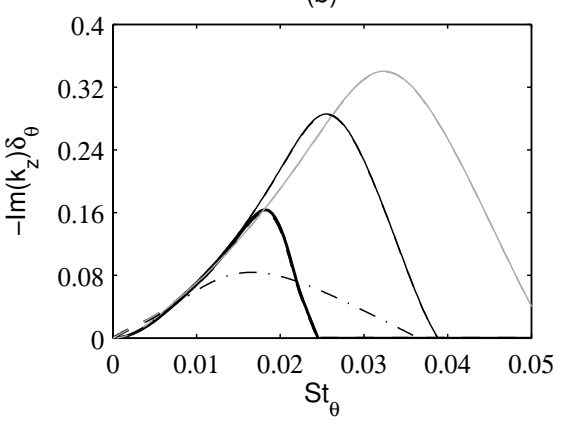

FiguRE 6. Representation (a) of the profiles of mean axial velocity $\left\langle u_{z}\right\rangle$ at $z=0.1 r_{0}$ and (b) of the instability growth rates $-\operatorname{Im}\left(k_{z}\right)$ obtained for the profiles using an inviscid linear stability analysis for modes $n_{\theta}=0$ for $\longrightarrow$ jetBL, — jetT1, $\longrightarrow$ jetT2 and $n_{\theta}=1$ for - - - jetBL, - - - jetT1, - - - jetT2, as a function of $\mathrm{St}_{\theta} ;-\cdots-$ results for a 2-D hyperbolic-tangent velocity profile with $\delta_{\theta}=0.0288 r_{0}$.

$\begin{array}{lcccc} & \mathrm{St}_{D} & \mathrm{St}_{\theta} & \mathrm{St}_{\omega} & \mathrm{St}^{+} \\ \text {jetBL } & 1.21 & 0.018 & 0.071 & 0.078 \\ \text { jetT1 } & 1.79 & 0.026 & 0.070 & 0.050 \\ \text { jetT2 } & 2.30 & 0.032 & 0.067 & 0.040\end{array}$

TABle 6. Peak Strouhal numbers $\mathrm{St}_{D}, \mathrm{St}_{\theta}, \mathrm{St}_{\omega}$ and $\mathrm{St}^{+}$of instability growth rates obtained using an inviscid linear stability analysis at $z=0.1 r_{0}$.

The resulting Strouhal numbers $\mathrm{St}_{\omega}=f \delta_{\omega} / u_{j}$ and $\mathrm{St}^{+}=f \nu / u_{\tau}^{2}$ are given in table 6 . As the boundary-layer shape factor $\mathrm{H}$ decreases, the latter varies from 0.078 for jetBL down to 0.040 for jetT2, whereas the former remains very close to 0.07 . Therefore, the frequency of the initial instability wave is primarily linked to the high-shear portion of the velocity profiles, as was noted by Fontaine et al. (2015).

The spectra of radial velocity fluctuations calculated at $r=r_{0}$ at $z=0.1 r_{0}, z=0.2 r_{0}$ and $z=0.4 r_{0}$ are represented in figure 7 as a function of $\mathrm{St}_{D}$. The peak diameter-based Strouhal numbers obtained from the mean flow profiles at $z=0.1 r_{0}$ using the linear stability analysis, provided in table 6, are also indicated. For all jets, a hump appears in the spectra, centered on a frequency moving slowly towards lower frequencies in the downstream direction, as for the separating boundary layer of Morris \& Foss (2003). The peak frequencies are in very good agreement with the linear stability results, especially in figure $7(\mathrm{~b})$ for $z=0.2 r_{0}$. Moreover, the hump rapidly grows, at a rate which is lowest for jetBL and highest for jetT2, as predicted by the instability amplification rates of figure $6(\mathrm{~b})$. Therefore, for the present initially disturbed jets, the flow development very near the nozzle is driven by the instability waves examined in this section.

\subsection{Shear-layer development}

\subsubsection{Vorticity snapshots}

Instantaneous fields of vorticity norm obtained down to $z=3.5 r_{0}$ and to $z=12 r_{0}$ are represented in figures $8(\mathrm{a}, \mathrm{c}, \mathrm{e})$ and $8(\mathrm{~b}, \mathrm{~d}, \mathrm{f})$, respectively. Very near the nozzle lip, in figures $8(\mathrm{a}, \mathrm{c}, \mathrm{e})$, the levels of vorticity are higher for jetT2 than for the two other jets due to the sharper boundary-layer profile. In that region, the turbulent structures are elongated in the downstream direction, which is characteristic of wall bounded flows. 
(a)

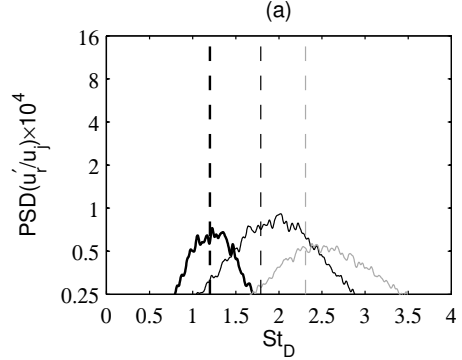

\section{(b)}

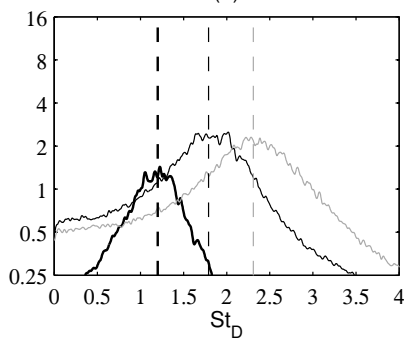

(c)

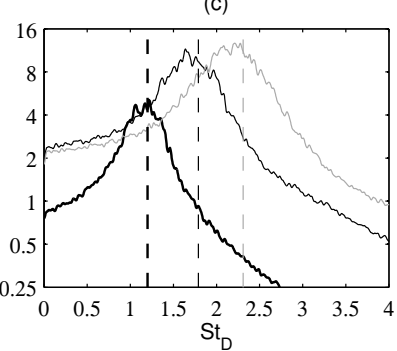

FIgURE 7. Power spectral densities of radial velocity fluctuations $u_{r}^{\prime}$ at $r=r_{0}$ at (a) $z=0.1 r_{0}$, (b) $z=0.2 r_{0}$ and (c) $z=0.4 r_{0}$, as a function of $\mathrm{St}_{D}:-$ jetBL, - jetT1, jetT2; and peak frequencies of instability growth rates obtained using an inviscid linear stability analysis at $z=0.1 r_{0}:---$ jetBL, -- jetT1, $-\cdots$ jetT2.

In the radial direction, their length scales are of the order of boundary-layer thickness for jetBL, but are much smaller for jetT1 and especially for jetT2. For the latter jet, in particular, strong levels of vorticity are only found around $r=r_{0}$. This is the case nearly down to $z=0.5 r_{0}$, in agreement with the persistence of the mean boundary-layer profile mentioned above. These results supports again that the initial shear-layer development is essentially related to the vorticity thickness of the velocity profile. Further away from the nozzle, the shear layers seem to roll up around $z=1.5 r_{0}$ for jetBL but earlier for jetT1 and jetT2, which is in line with the instability amplification rates of the previous section. Then, they exhibit typical features of turbulent mixing layers. Finally, in figures $8(\mathrm{~b}, \mathrm{~d}, \mathrm{f})$, the mixing layers appear to be fully developed for $z \gtrsim 4 r_{0}$. However, they spread faster for jetBL than for the two other jets. The presence of large-scale structures resembling the coherent structures of the flow visualizations of Brown \& Roshko (1974) is also more obvious for the laminar boundary-layer profile than for the non-laminar profiles. Similar effects of the exit velocity profile on the organized structures in the shear layers of jets were recently revealed by the experiments of Zaman (2017) using the ASME and the conical nozzles. It should be reminded that the definition of coherent structures may vary from one researcher to another. In this work, following Hussain (1986) and Fieldler (1988), they refer to regions of correlated and concentrated vorticity, of size comparable to the transverse length scale of the shear layer, which are spatially isolated from each other and show similarity with the corresponding structures of the (preceding) laminarturbulent transition.

\subsubsection{Flow field properties}

The variations of the shear-layer momentum thickness are represented over $0 \leq z \leq 6 r_{0}$ in figure $9(\mathrm{a})$ and over $0 \leq z \leq 15 r_{0}$ in figure $9(\mathrm{~b})$. The spreading rates $\mathrm{d} \delta_{\theta} / \mathrm{d} z$ are also shown in figure 9 (a). The differences are significant between jetBL and jetT1 with boundary-layer profiles with $\mathrm{H}=2.29$ and $\mathrm{H}=1.96$, but they are rather weak between the two transitional cases with $\mathrm{H}=1.96$ and $\mathrm{H}=1.71$. For $z \leq 3 r_{0}$, the mixing layers develop faster for jetT1 and jetT2 than for jetBL. This can be due to the higher growth rates of the jet initial instability waves as the shape factor $\mathrm{H}$ decreases, highlighted in figure 6. Farther downstream, in contrast, the mixing layers spread most rapidly for jetBL, which was suggested by the vorticity fields of figure 8 , but has no evident cause at first sight. In this region, a better agreement with the measurements of Fleury (2006) and Castelain (2006) for jets at $\mathrm{M}=0.9$ and $\mathrm{Re}_{D} \simeq 10^{6}$, undoubtedly initially turbulent, is obtained for the jets with non-laminar boundary-layer profiles. Furthermore, for jetBL, the shear-layer spreading rate increases monotonically with the axial distance up to values 
(a)

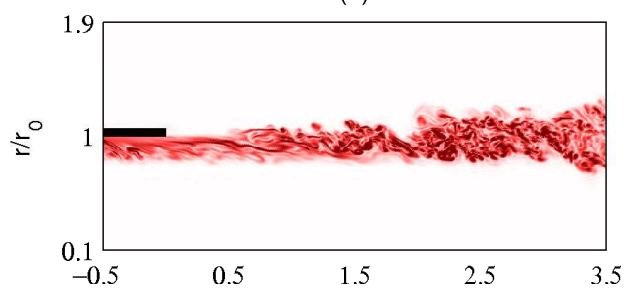

(c)

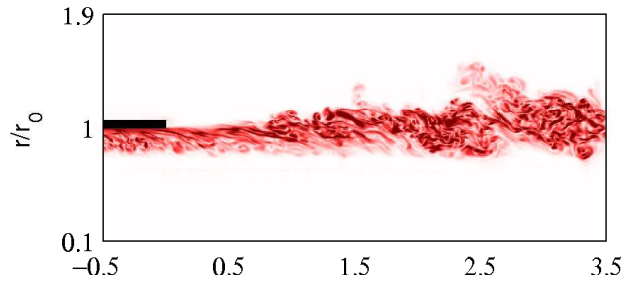

(e)

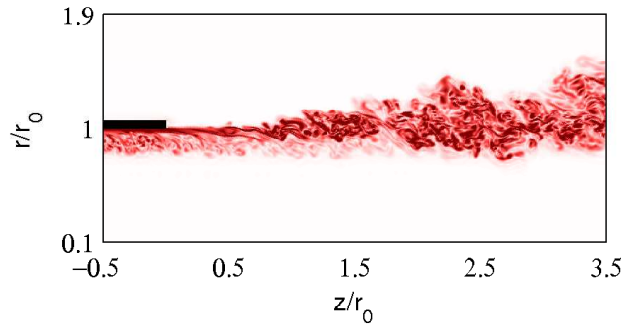

(b)

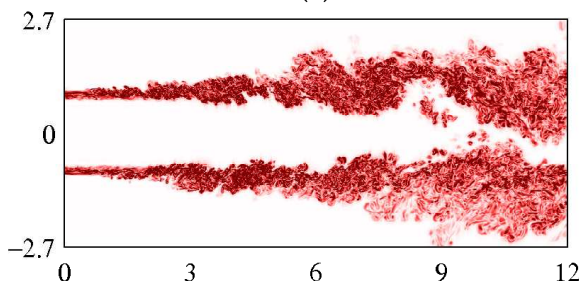

(d)

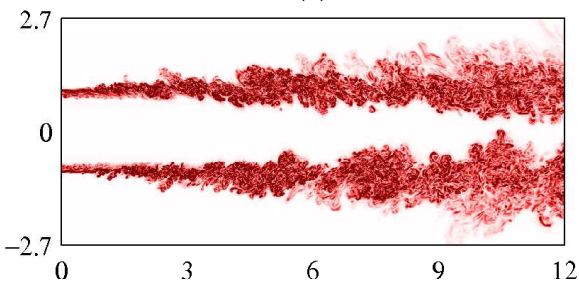

(f)

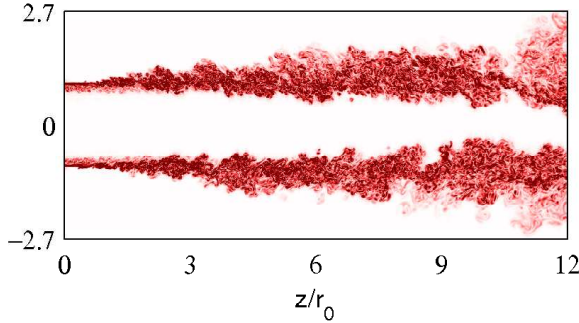

Figure 8. (Colour available at journals. cambridge.org/flm) Snapshots in the $(z, r)$ plane of vorticity norm $|\boldsymbol{\omega}|$ for (a) jetBL, (b) jetT1 and (c) jetT2. The color scales range from 0 up to $(\mathrm{a}, \mathrm{c}, \mathrm{e}) 18 u_{j} / r_{0}$ and $(\mathrm{b}, \mathrm{d}, \mathrm{f}) 9 u_{j} / r_{0}$, from white to red.

(a)

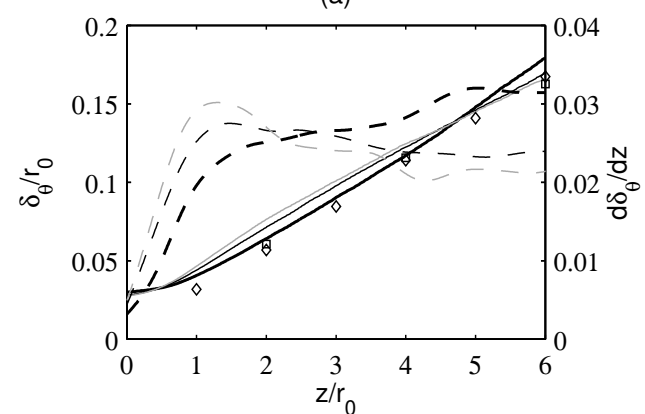

(b)

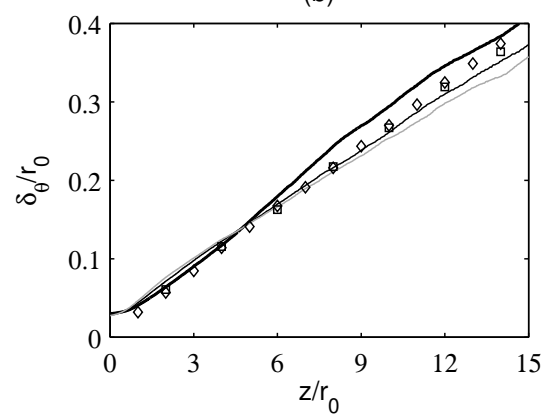

FiguRE 9. Variations of shear-layer momentum thickness $\delta_{\theta}$ for

jetBL, jetT1 and $\longrightarrow$ jetT2 and of spreading rate $\mathrm{d} \delta_{\theta} / \mathrm{d} z$ for $-\ldots$ jetBL, -- jetT1 and -- jetT2; measurements for isothermal jets at $\mathrm{M}=0.9: \diamond$ Fleury $(2006)$ at $\operatorname{Re}_{D}=7.7 \times 10^{5}$ and $\square$ Castelain (2006) at $\operatorname{Re}_{D}=10^{6}$.

around 0.030 at $z=5 r_{0}$. For jetT1 and jetT2, on the contrary, they reach peak values of 0.0275 at $z=1.3 r_{0}$ and of 0.030 at $z=1.5 r_{0}$, respectively, and do not exceed values of 0.024 for $z \geq 4 r_{0}$.

In order to illustrate the change of the mean flow profiles in the region of boundarylayer/mixing-layer transition, the profiles of mean axial velocity at $z=0.8 r_{0}, z=1.6 r_{0}$ 
(a)

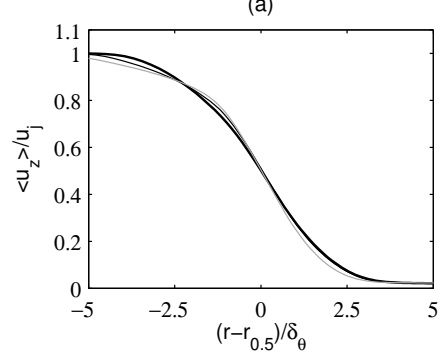

(b)

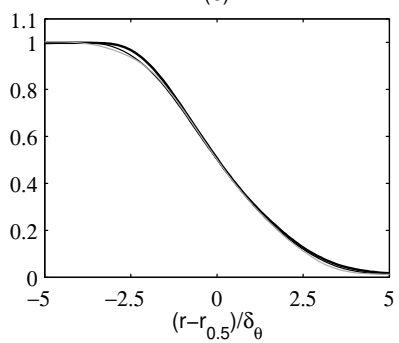

(c)

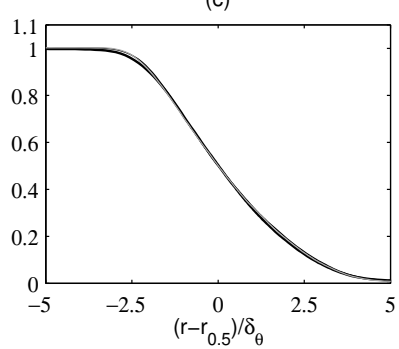

FIGURE 10. Radial profiles of mean axial velocity $\left\langle u_{z}\right\rangle$ at (a) $z=0.8 r_{0}$, (b) $z=1.6 r_{0}$ and (c) $z=3.2 r_{0}:-$ jetBL, $\longrightarrow$ jetT1, $\longrightarrow$ jetT2.

and $z=3.2 r_{0}$ are provided in figure 10 . The radial distances are normalized by the local shear-layer momentum thicknesses, which, however, are nearly the same in the present jets at $z=0.8 r_{0}$ and $z=3.2 r_{0}$, and only vary from $0.054 r_{0}$ in jetBL up to $0.065 r_{0}$ in jetT2 at $z=1.6 r_{0}$. At $z=0.8 r_{0}$, corresponding to $z=28 \delta_{\theta}(0)$, the velocity profiles differ significantly. This is particularly the case for their high-speed portions, which still bear strong similarities with the nozzle-exit profiles. The latter result is consistent with that obtained for a turbulent boundary layer at $z=29 \delta_{\theta}(0)$ in the experiments of Morris $\&$ Foss (2003). Farther away from the nozzle, the mean velocity profiles are very close to each other at $z=1.6 r_{0}$ and almost superimposed at $z=3.2 r_{0}$, and exhibit no clear reminiscence of the boundary-layer profiles.

The rms values of axial and radial velocity fluctuations at $r=r_{0}$ are displayed down to $z=15 r_{0}$ in figure 11 . They follow trends which are similar to those for the mixing-layer spreading rate. Just downstream of the nozzle, they increase more rapidly for jetT1 and jetT2 than for jetBL, thus reaching peaks around $z=r_{0}$ in the former case, but $z=5 r_{0}$ in the latter. In addition, the levels are lower for the transitional boundary-layer profiles. This is true for the peak levels in the jets, given in table 7 , which are equal, for $u_{z}^{\prime}$ and $u_{r}^{\prime}$ for instance, to approximately $0.157 u_{j}$ and $0.12 u_{j}$ for jetT1 and jetT2, but to $0.174 u_{j}$ and $0.131 u_{j}$ for jetBL. The difference in turbulence intensity is also significant down to $z=15 r_{0}$, which is roughly the position of the end of the jet potential core. Therefore, the effects of the exit boundary-layer profile on the turbulence in the mixing layers last far downstream of the nozzle, despite, notably, the nearly identical mean flow profiles obtained at $z=3.2 r_{0}$ in figure 10 (c). Finally, as for the shear-layer momentum thickness, the results for the jets with non-laminar mean velocity profiles better agree with the measurements of Fleury (2006) and Castelain (2006) than those for jetBL.

Comparisons between numerical and experimental data may only be fully relevant for identical upstream flow conditions. It can however be mentioned that in the similarity region of an axisymmetric mixing layer, initially with $\operatorname{Re}_{\theta}=349, u_{e}^{\prime} / u_{j}=6.18 \%$ and $\mathrm{H}=2.47$, Hussain \& Zedan (1978b) obtained a spreading rate of 0.0294 and a peak axial turbulence intensity of $16.7 \%$, which are both comparable to the values reached in jetBL. Moreover, the changes undergone by the mixing layers of the present jets as the nozzleexit velocity profile deviates from a laminar profile, namely a slower growth and weaker velocity fluctuations, correspond to those observed experimentally when initially laminar shear layers are tripped and become initially turbulent (Hill et al. 1976; Browand \& Latigo 1979; Husain \& Hussain 1979). They also resemble the changes induced by increasing the exit turbulence levels only (Hussain \& Zedan 1978a; Bogey et al. 2012b).

Finally, the skewness factors of the axial and radial velocity fluctuations at $r=r_{0}$ are represented in figure 12 . In the vicinity of the nozzle exit, in all cases, they differ 
(a)

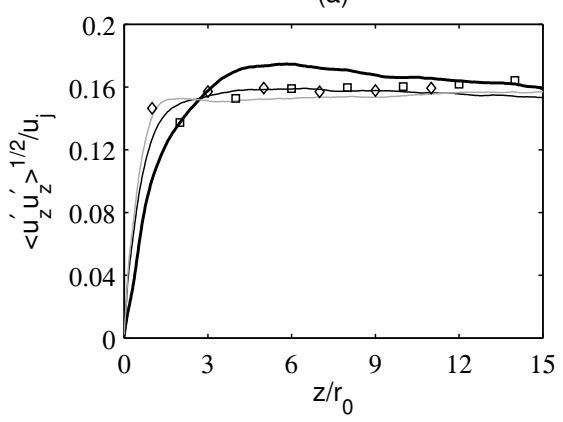

(b)

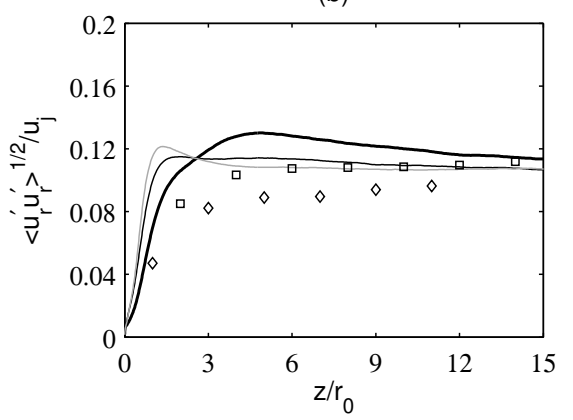

FiguRE 11. Variations of the rms values of (a) axial and (b) radial velocity fluctuations $u_{z}^{\prime}$ and $u_{r}^{\prime}$ at $r=r_{0}$ : — jetBL, - jetT1, - jetT2; peak values measured in isothermal jets at $\mathrm{M}=0.9 \mathrm{~s} \diamond$ Fleury $(2006)$ at $\operatorname{Re}_{D}=7.7 \times 10^{5}$ and $\square$ Castelain (2006) at $\operatorname{Re}_{D}=10^{6}$.

$\begin{array}{lcccc} & \left\langle u_{z}^{\prime 2}\right\rangle^{1 / 2} / u_{j} & \left\langle u_{r}^{\prime 2}\right\rangle^{1 / 2} / u_{j} & \left\langle u_{\theta}^{\prime 2}\right\rangle^{1 / 2} / u_{j} & \left\langle u_{r}^{\prime} u_{z}^{\prime}\right\rangle^{1 / 2} / u_{j} \\ \text { jetBL } & 17.4 \% & 13.1 \% & 14.5 \% & 10.6 \% \\ \text { jetT1 } & 15.9 \% & 11.7 \% & 13.5 \% & 9.6 \% \\ \text { jetT2 } & 15.5 \% & 12.3 \% & 14.0 \% & 9.9 \%\end{array}$

TABLE 7. Peak turbulence intensities in the jets.

(a)

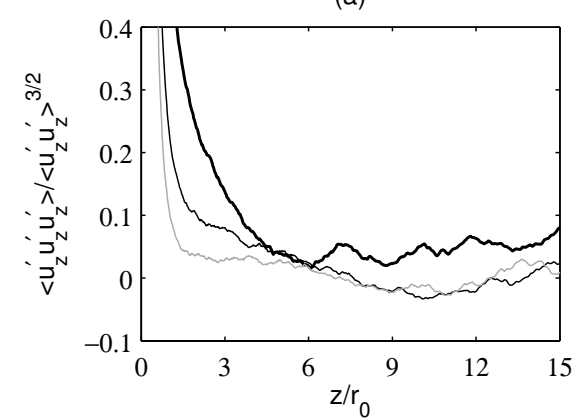

(b)

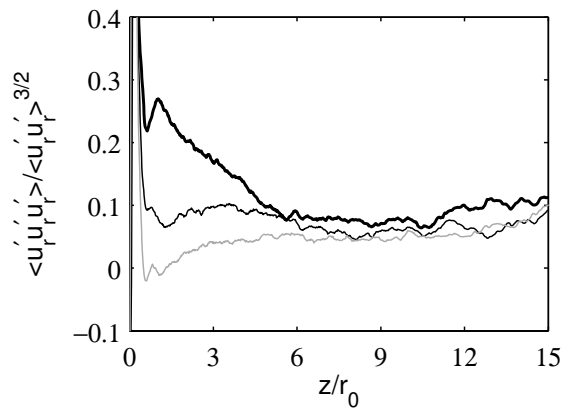

Figure 12. Variations of the skewness values of (a) axial and (b) radial velocity fluctuations

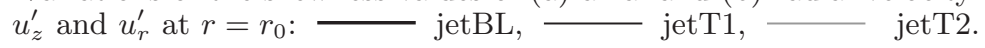

appreciably from zero, which is expected at the interface between the highly-disturbed shear layers and the ambient medium. Their positive values are due to the sudden eruptions of high-velocity fluid at the outer edge of the mixing layers. For jetT1 and jetT2, the skewness factors rapidly decrease, whereas they remain greater than 0.1 down to $z=4 r_{0}$ for jetBL. This can be related to the slower initial development of the shear layers in the latter case. Farther downstream, for $z \geq 6 r_{0}$, the skewness factors, albeit much lower than previously, are still higher for jetBL than for the other jets. Given the links between velocity skewness and large-scale vortices in free shear flows (Yule 1978), this result suggests the presence of stronger coherent structures in the first jet.

\subsubsection{Instability waves and velocity spectra}

Some results of the inviscid linear stability analysis carried out, as reported in section 2.4, from the LES mean flow fields between $z=0.02 r_{0}$ and $z=5 r_{0}$ are provided in 

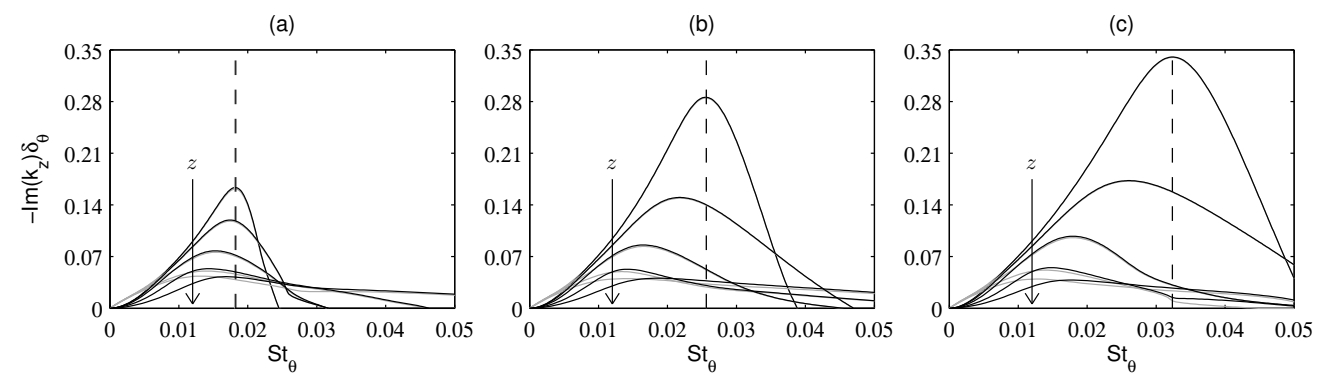

FIGURE 13. Representation of the instability growth rates $-\operatorname{Im}\left(k_{z}\right)$ obtained using an inviscid linear stability analysis at $z=0.1 r_{0}, 0.4 r_{0}, 0.8 r_{0}, 1.6 r_{0}$ and $3.2 r_{0}$ for $-n_{\theta}=0$ and $\longrightarrow n_{\theta}=1$ for (a) jetBL, (b) jetT1 and (c) jetT2, as a function of $\mathrm{St}_{\theta}$; - - peak frequencies at $z=0.1 r_{0}$.

order to investigate the properties of the instability waves, and their variations in the axial direction, during the boundary-layer/mixing-layer transition and further downstream, They will help us to identify the possible cause of the differences between the shear-layer developments.

The instability amplification rates $-\operatorname{Im}\left(k_{z}\right) \delta_{\theta}$ calculated for $n_{\theta}=0$ and $n_{\theta}=1$ at $z=$ $0.1 r_{0}, 0.4 r_{0}, 0.8 r_{0}, 1.6 r_{0}$ and $3.2 r_{0}$ are represented in figure 13 as a function of the Strouhal number $\mathrm{St}_{\theta}$. The curves obtained for the two azimuthal modes are nearly superimposed on each other except for $z=3.2 r_{0}$, where lower unstable frequencies are found for $n_{\theta}=1$ than for $n_{\theta}=0$ due to the mixing-layer thicknesses of $\delta_{\theta} \simeq 0.1 r_{0}$ at this location (Michalke 1984). As the distance from the nozzle exit increases, the amplification curves change appreciably in level and shape for all jets. For jetBL, the instability growth rates are lower, and the ranges of unstable frequencies broaden. However, the peak Strouhal numbers, equal to $\mathrm{St}_{\theta}=0.018$ at $z=0.1 r_{0}$, do not vary much with the axial position. For jetT1 and jetT2 with non-laminar boundary-layer profiles, the changes with the distance from the nozzle are more important. The reduction of the growth rates is stronger and, above all, the peak Strouhal numbers $\mathrm{St}_{\theta}$, of 0.026 for jetT1 and of 0.032 for jetT2 at $z=0.1 r_{0}$, decrease significantly. At $z=3.2 r_{0}$, finally, the amplification curves are the nearly the same for the three jets, which is not surprising given the very similar velocity profiles of figure 10 (c).

In order to highlight their variations downstream of the nozzle, the peak Strouhal numbers $\mathrm{St}_{\theta}$ of the instability growth rates are plotted in figure 14 (a) between $z=0.02 r_{0}$ and $z=3.5 r_{0}$. The values obtained for $n_{\theta}=0$ and $n_{\theta}=1$ are identical to each other down to $z \simeq r_{0}$, and then gradually diverge due to the thickening of the mixing layer, yielding $\mathrm{St}_{\theta} \simeq 0.018$ for $n_{\theta}=0$ and $\mathrm{St}_{\theta} \simeq 0.014$ for $n_{\theta}=1$ at $z=3.5 r_{0}$ in all cases. More interestingly, strong discrepancies appear in the vicinity of the nozzle exit between the three jets. In that region, for jetBL, the peak Strouhal numbers do not change much with the axial distance and remain close to a value of $\mathrm{St}_{\theta}=0.018$ corresponding roughly to the Strouhal numbers emerging farther downstream in the mixing layers. For jetT1 and jetT2, on the contrary, they rapidly decrease during the changeover from a boundarylayer profile to a mixing-layer profile, from values of the order of or higher than 0.03 at $z=0.02 r_{0}$ down to values lower than 0.02 at $z \simeq 0.6 r_{0} \simeq 20 \delta_{\theta}(0)$. These variations of $\mathrm{St}_{\theta}$ are in very good agreement with the experimental data of Morris \& Foss (2003) for a turbulent boundary layer.

As in the study mentioned above, a scaling with the local shear-layer vorticity thickness is applied to the peak frequencies of the instability growth rates. The resulting Strouhal numbers $\mathrm{St}_{\omega}$ are shown in figure $14(\mathrm{~b})$ between $z=0.02 r_{0}$ and $z=3.5 r_{0}$. For the 
(a)

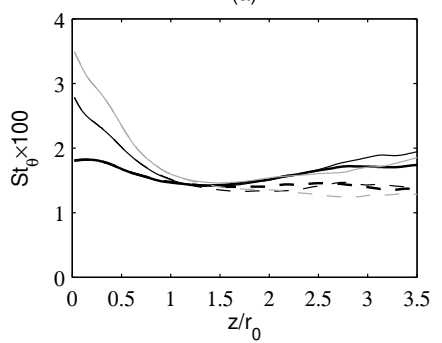

(b)

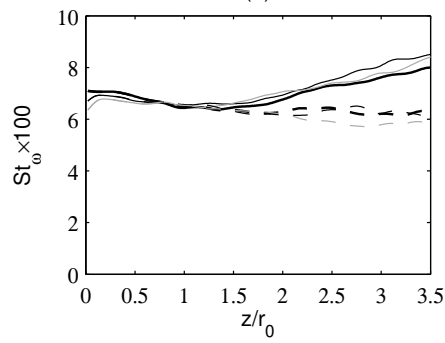

(c)

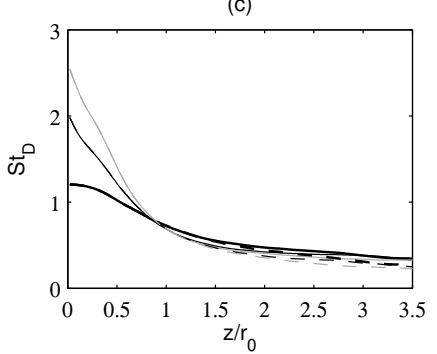

Figure 14. Axial variations of the peak Strouhal numbers (a) $\mathrm{St}_{\theta}$, (b) $\mathrm{St}_{\omega}$ and (c) $\mathrm{St}_{D}$ of instability growth rates obtained for - jetBL, - jetT1, - jetT2 for $n_{\theta}=0$; $--{ }_{-},---,--$corresponding results for $n_{\theta}=1$.

(a)

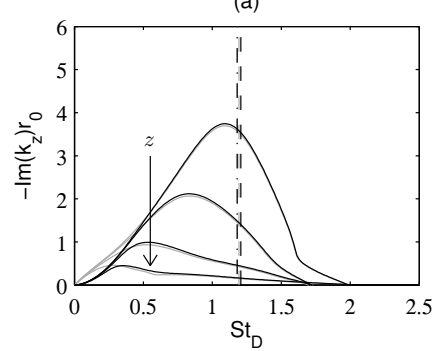

FURE $15 \cdot$ Representation of $1.6 r_{0}$ and $3.2 r_{0}$ as a function of $\mathrm{St}_{D}$ and of the peak frequencies at $--z=0.1 r_{0}$ and - $0.2 r_{0}$ obtained using an inviscid linear stability analysis for $n_{\theta}=0$ for (a) jetBL, (b) jetT1 and $(\mathrm{c})$ jetT2; $\ldots, \ldots$ and $\ldots \ldots$ corresponding results for $n_{\theta}=1$.

(b)

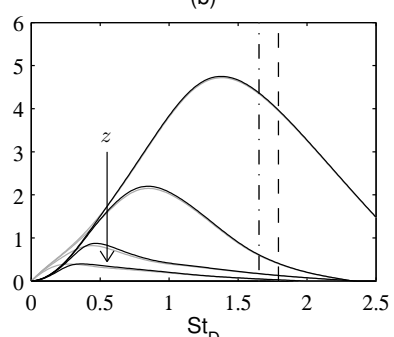

(c)

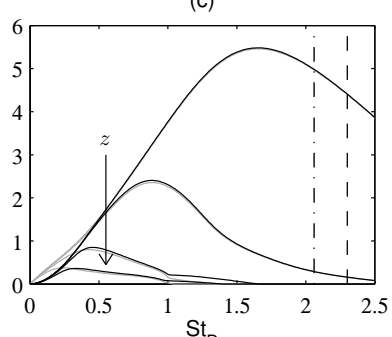

present jets, they are very close to each other at any of the locations considered. This is particularly true, despite the different boundary-layer profiles, near the nozzle, where Strouhal numbers $\mathrm{St}_{\omega} \simeq 0.07$ are continuously found between $z=0.02 r_{0}$ and $z \simeq 2 r_{0}$. Therefore, for a given mean flow profile, the peak frequency of the instability waves is only fixed by the maximum velocity gradient.

The variations of the most unstable Strouhal numbers $\mathrm{St}_{\theta}$ downstream of the nozzle do not reflect those of the most unstable frequencies because of the increase of the shearlayer momentum thickness in the axial direction. For that reason, the instability growth rates $-\operatorname{Im}\left(k_{z}\right) r_{0}$ obtained for $n_{\theta}=0$ and $n_{\theta}=1$ at $z=0.4 r_{0}, 0.8 r_{0}, 1.6 r_{0}$ and $3.2 r_{0}$ are re-plotted in figure 15 as a function of the diameter-based Strouhal number $\mathrm{St}_{D}$. The peak Strouhal numbers $\mathrm{St}_{D}$ are also represented in figure 14(c) between $z=0.02 r_{0}$ and $z=3.5 r_{0}$. As the distance from the nozzle increases, they move to lower values due to the shear-layer thickening. During the initial stage of flow development between the nozzle exit and $z \simeq 0.6 r_{0}$, the frequency decrease is however much more pronounced for jetT1 and jetT2 than for jetBL. In their linear stability analyses, Brès et al. (2018) recently noted, as in this work, that downstream of the nozzle the range of the unstable frequencies are more quickly reduced for their initially turbulent jet than for their initially laminar jet with thicker exit boundary layer. They attributed this to the fact that the instability waves in the near-nozzle region grow at a higher rate in the first jet because of the faster shear-layer spreading in this case. On the basis of the present results, this appears to be also strongly linked to the difference in peak instability frequency between laminar and non-laminar boundary-layer profiles.

The dependence of the range of the unstable frequencies on the boundary-layer profiles 

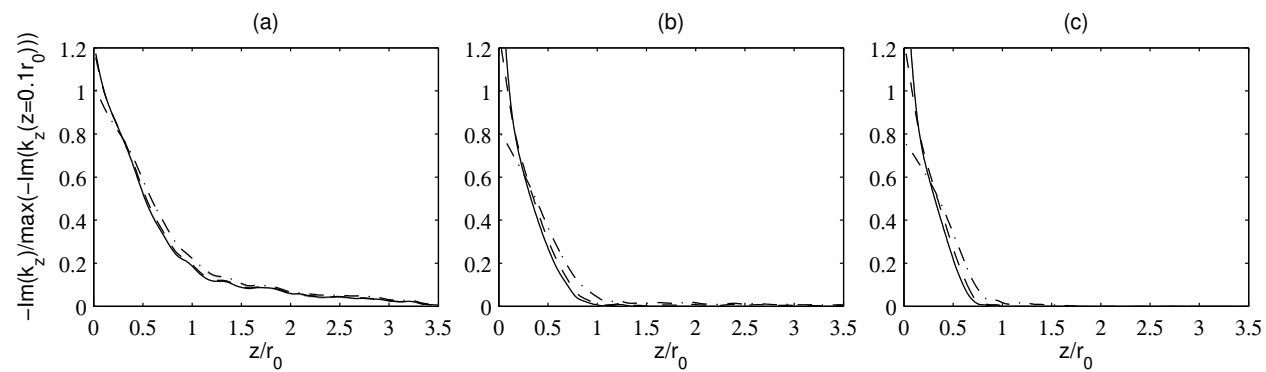

FIGURE 16. Axial variations of the instability growth rates obtained at the peak Strouhal numbers $\mathrm{St}_{D}$ at $-z=0.1 r_{0},---z=0.2 r_{0}$ and $-\cdot-\cdot z=0.4 r_{0}$ for $n_{\theta}=0$ for (a) jetBL, (b) jetT1 and (c) jetT2, normalized by the maximum growth rates at $z=0.1 r_{0}$; ,$-\ldots$ and $\ldots-\ldots$ corresponding results for $n_{\theta}=1$.

has substantial effects on the spatial evolution of the instability waves developing downstream of the nozzle. Examine, for instance, the peak frequencies obtained at $z=0.1 r_{0}$ in figure 15. The more non-laminar the boundary-layer profile, the earlier they leave the range of the unstable frequencies. The growth rates calculated between $z=0.02 r_{0}$ and $z=3.5 r_{0}$ for the peak frequencies at $z=0.1 r_{0}, 0.2 r_{0}$ and $0.4 r_{0}$, chosen to cover the frequency range of the initial instability waves, are also represented in figure 16 . In all cases, they sharply decrease downstream of the nozzle. However, they remain appreciable down to $z \simeq 3.5 r_{0} \simeq 125 \delta_{\theta}(0)$ in figure 16 (a) for jetBL, whereas they become negligible or negative as early as $z \simeq r_{0} \simeq 35 \delta_{\theta}(0)$ in figures $16(\mathrm{~b}, \mathrm{c})$ for jetT1 and jetT2. As a result, the instability waves developing very near the nozzle continue to be amplified, even at a low rate, over a relatively large axial distance for the laminar boundary-layer profile, whereas they are rapidly damped for the non-laminar profiles.

Velocity spectra computed in the mixing layers are discussed in light of the results of the linear stability analysis. First, the spectra of radial velocity fluctuations obtained at $r=r_{0}$ at $z=0.8 r_{0}, 1.6 r_{0}, 3.2 r_{0}, 4.8 r_{0}, 6.4 r_{0}$ and $10 r_{0}$ are represented in figure 17 as a function of the Strouhal number $\mathrm{St}_{D}$, along with the peak frequencies of instability growth rates at $z=0.1 r_{0}$. At $z=0.8 r_{0}$, in figure $17(\mathrm{a})$, the spectra resemble those of figure 7 acquired farther upstream. They are dominated by humps associated with the initial instability waves, peaking at frequencies slightly lower than those predicted at $z=0.1 r_{0}$ due to the shear-layer thickening. As the distance from the nozzle increases, in all cases, the humps diminish and eventually vanish as turbulence develops in the mixing layers. However, for jetBL, the hump remains noticeable at $z=4.8 r_{0}$ in figure $17(\mathrm{~d})$, whereas they cannot be observed at $z=3.2 r_{0}$ in figure $17(\mathrm{c})$ for jetT1 and jetT2. This discrepancy can be explained by the linear stability analysis, indicating a longer persistence of the initial instability waves for the laminar boundary layer than for the transitional ones. Farther downstream, at $z=6.4 r_{0}$ and $z=10 r_{0}$ in figures $17(\mathrm{e}, \mathrm{f})$, the spectra are all broadband, but significant differences appear at low frequencies. More precisely, the levels are higher for jetBL than for jetT1 and jetT2 at $\mathrm{St}_{D} \lesssim 1$. Therefore, in the jet with a laminar boundary layer, the initial instability components last over a larger distance, but also lead to stronger large-scale structures in the mixing layers after having disappeared. These results are in line with the comments on coherent structures made previously from the vorticity fields and the skewness factors at $r=r_{0}$, and with the visualizations of Zaman (2017) for initially nominally laminar jets.

In order to explore the azimuthal distribution of the flow disturbances, the spectra of radial velocity fluctuations at $r=r_{0}$ at $z=0.8 r_{0}, 3.2 r_{0}$ and $10 r_{0}$ are depicted in figure 18 as a function of mode $n_{\theta}$. At the first location, in figure 18(a), the spectra have 


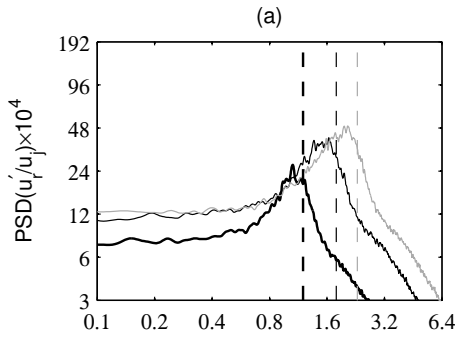

(d)

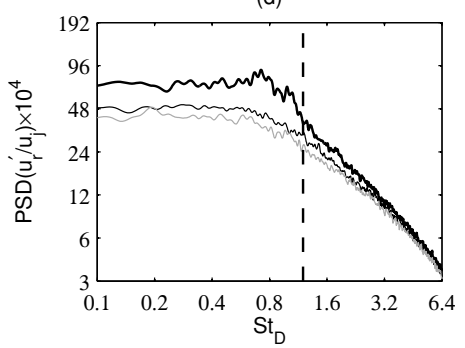

(b)

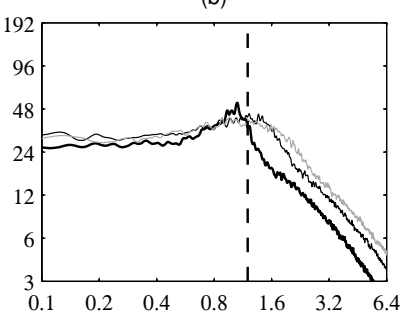

(e)

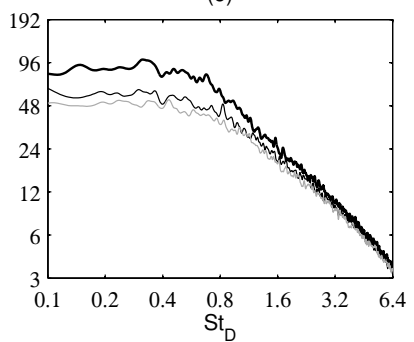

(c)

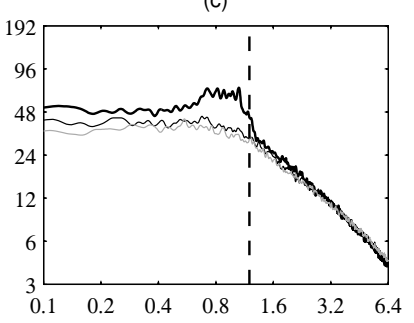

(f)

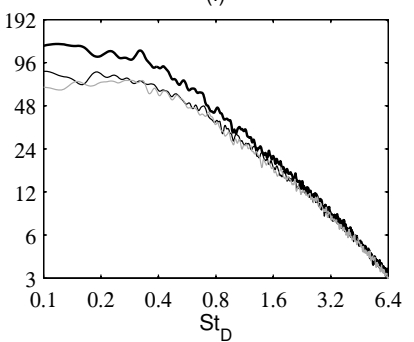

Figure 17. Power spectral densities of radial velocity fluctuations $u_{r}^{\prime}$ at $r=r_{0}$ at (a) $z=0.8 r_{0}$, (b) $z=1.6 r_{0}$, (c) $z=3.2 r_{0}$, (d) $z=4.8 r_{0}$, (e) $z=6.4 r_{0}$ and (f) $z=10 r_{0}$ as a function of $\mathrm{St}_{D}$ : $\longrightarrow$ jetBL, - jetT1, $\longrightarrow$ jetT2; peak frequencies of instability growth rates obtained using an inviscid linear stability analysis at $z=0.1 r_{0}$ : - - - jetBL, - - jetT1, - - - jetT2.

nearly identical shapes over the whole range of modes considered. Since the azimuthal velocity spectra at the nozzle exit are also close to each other in figures 4(b) and 5(b), the mechanisms at play between $z=0$ and $z=0.8 r_{0}$ are of the same nature in the three jets. The levels are highest for jetT2 and lowest for jetBL, and for a given jet, they are maximum for the axisymmetric mode, remain strong up to modes $n_{\theta}=3$ or 4 , and then sharply decrease for higher modes. These trends are consistent with the features of the instability waves initially growing in the shear layers, namely higher amplification rates for a more turbulent nozzle-exit boundary layer, and very similar rates for the first five azimuthal modes (Brès et al. 2018). Farther downstream, at $z=3.2 r_{0}$ and $z=10 r_{0}$ in figure $18(\mathrm{~b}, \mathrm{c})$, the spectra are superimposed for $n_{\theta} \geq 16$, but the levels are higher for jetBL than for jetT1 and jetT2 at lower mode numbers. The difference in level is largest for $n_{\theta} \leq 2$ at $z=3.2 r_{0}$, which may be related to the presence of instability components at this position for jetBL, and for $n_{\theta} \leq 5$ at $z=10 r_{0}$. The intense large-scale structures in the mixing layers of jetBL revealed by the spectra of figures 17 (c-f) are consequently significantly correlated in the azimuthal direction.

Finally, the spectra of radial velocity fluctuations at $r=r_{0}$ at $z=0.8 r_{0}, 3.2 r_{0}$ and $10 r_{0}$ for mode $n_{\theta}=1$ are displayed in figure 19 as a function of $\mathrm{St}_{D}$. For brevity, only the results for $n_{\theta}=1$ are reported, but those obtained for the other first azimuthal modes are very similar. As in figures $17(\mathrm{a}, \mathrm{c}, \mathrm{f})$, humps associated with the initial instability waves dominate at $z=0.8 r_{0}$, the hump still appears only for jetBL at $z=3.2 r_{0}$, and the low-frequency components are stronger for jetBL than for the other jets at $z=10 r_{0}$. The instability waves however emerge more clearly in the present case than in the spectra computed from the full velocity fields. Compared to the broadband levels, indeed, their peak levels are more than two decades higher in figure 19(a), whereas they are 3-4 times higher in figure $17(\mathrm{a})$. 
(a)

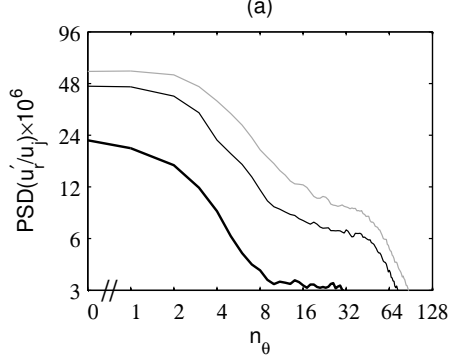

(b)

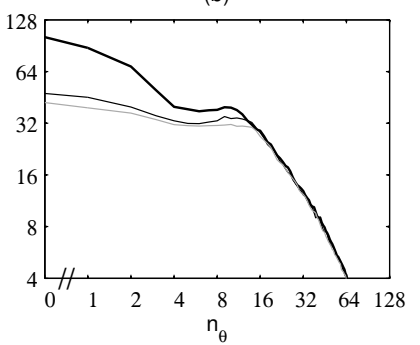

(c)

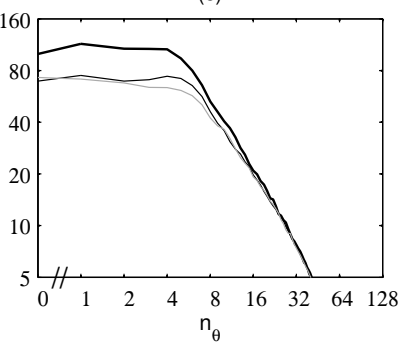

FIGURE 18. Power spectral densities of radial velocity fluctuations $u_{r}^{\prime}$ at $r=r_{0}$ at (a) $z=0.8 r_{0}$, (b) $z=3.2 r_{0}$ and (c) $z=10 r_{0}$, as a function of mode $n_{\theta}:-$ jetBL, - jetT1, jetT2.

(a)

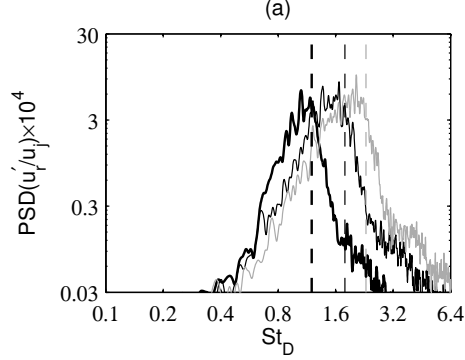

(b)

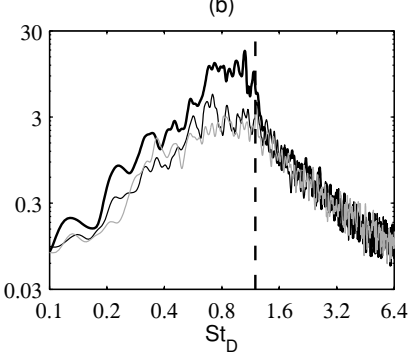

(c)

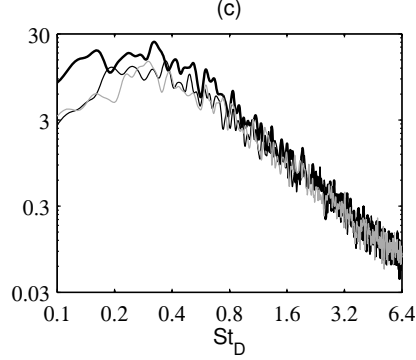

FIgURE 19. Power spectral densities for mode $n_{\theta}=1$ of radial velocity fluctuations $u_{r}^{\prime}$ at $r=r_{0}$ at (a) $z=0.8 r_{0}$, (b) $z=3.2 r_{0}$ and (c) $z=10 r_{0}$, as a function of $\operatorname{St}_{D}:-$ jetBL,

jetT1, — jetT2; peak frequencies of instability growth rates obtained using an inviscid linear stability analysis at $z=0.1 r_{0}:--$ jetBL, -- jetT1, -- jetT2.

\subsection{Jet development}

\subsubsection{Vorticity snapshots}

Snapshots of the vorticity norm obtained from the nozzle exit down to $z=25 r_{0}$ are provided in figure 20. Overall, they look like each other, and display, from upstream to downstream, the growth of the turbulent mixing layers, the closing of the jet potential cores and the regions of developed jet flows. Large-scale coherent structures may also be seen in the shear layers, for instance at $z \simeq 11 r_{0}$ for jetBL and at $z \simeq 12 r_{0}$ for jetT2. As the shape factor of the exit boundary-layer profile decreases, the mixing layers visibly merge later, as expected given the reduction in shear-layer spreading rate noted in previous section. As a result, the end of the potential core is located around $z=13 r_{0}$ in figure 20(a) for the laminar boundary-layer profile, but around $z=15 r_{0}$ in figure 20(c) for the transitional profile with $\mathrm{H}=1.71$.

\subsubsection{Flow field properties}

The variations of the centerline mean axial velocity are presented in figure 21 . In figure 21(a), as the nozzle-exit boundary-layer profile changes from laminar to turbulent, the jet flow develops more slowly. The potential core thus ends at $z_{c}=12.4 r_{0}$ for jetBL, $14.8 r_{0}$ for jetT1 and $15.6 r_{0}$ for jetT2, as indicated in table 8 , where $z_{c}$ is defined such as $\left\langle u_{z}\right\rangle\left(z_{c}\right)=0.95 u_{j}$ at $r=0$. Even if the comparisons must be taken with care due to the moderate Reynolds number and the thick initial shear layers of the present jets, this leads to a better agreement with the measurements of Lau et al. (1979) and Fleury et al. (2008) for jets at $\mathrm{M}=0.9$ and $\mathrm{Re}_{D} \simeq 10^{6}$ plotted in the figure. Downstream of the potential core, the centerline velocity seems to decay at a similar rate in three jets. 
(a)

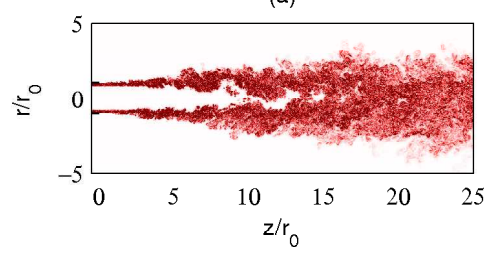

(b)

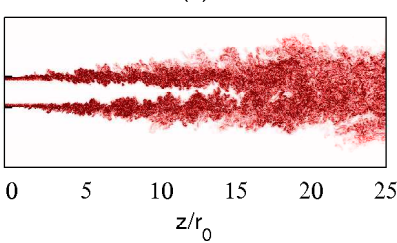

(c)

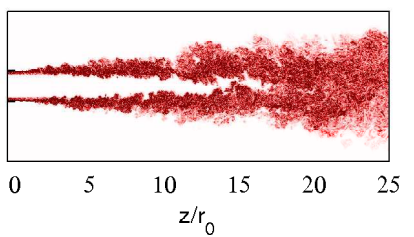

Figure 20. (Colour available at journals.cambridge.org/flm) Snapshots in the $(z, r)$ plane of vorticity norm $|\boldsymbol{\omega}|$ for (a) jetBL, (b) jetT1 and (c) jetT2. The color scale ranges from 0 up to $5.5 u_{j} / r_{0}$, from white to red.

(a)

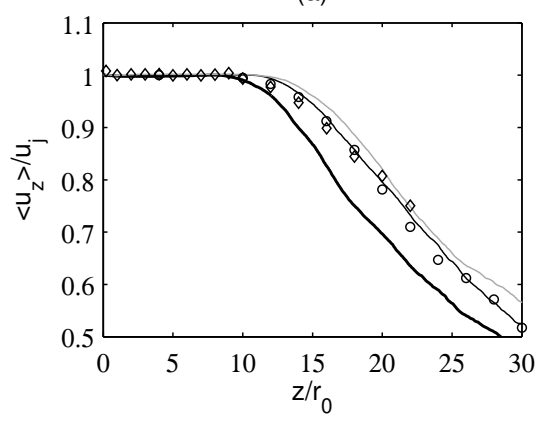

(b)

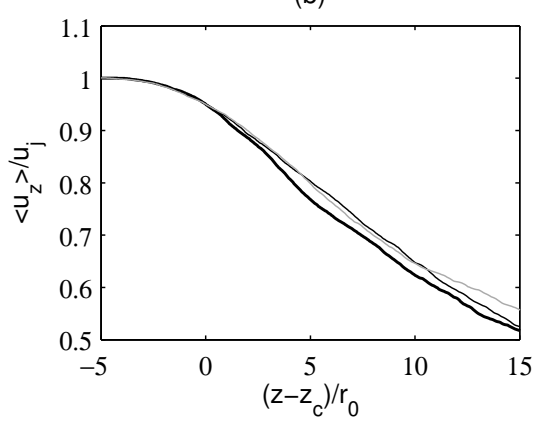

FIGURE 21. Variations of centerline mean axial velocity $\left\langle u_{z}\right\rangle$ as a function of (a) $z$ and (b) $z-z_{c}$ : jetBL, $\longrightarrow$ jetT1, jetT2; measurements for isothermal jets at $\mathrm{M}=0.9$ : $\circ$ Lau et al. $(1979)$ at $\operatorname{Re}_{D}=10^{6}$ and $\diamond$ Fleury et al. $(2008)$ at $\operatorname{Re}_{D}=7.7 \times 10^{5}$.

$\begin{array}{cccc} & z_{c} / r_{0} & \left\langle u_{z}^{\prime 2}\right\rangle^{1 / 2} / u_{j} & \left\langle u_{r}^{\prime 2}\right\rangle^{1 / 2} / u_{j} \\ \text { JetBL } & 12.4 & 14.3 \% & 11 \% \\ \text { JetT1 } & 14.8 & 12.9 \% & 10.1 \% \\ \text { JetT2 } & 15.6 & 13.7 \% & 10.3 \%\end{array}$

TABLE 8. Axial position of the end of the potential core $z_{c}$ and peak rms values of velocity fluctuations $u_{z}^{\prime}$ and $u_{r}^{\prime}$ on the jet axis.

According to figure 21(b), however, the decay rate is slightly lower for jetT1 and jetT2 than for jetBL.

The centerline rms values of axial velocity fluctuations are shown in figure $22(\mathrm{a})$. As for the mean flow profiles, the differences are significant between jetBL and the two jets with transitional boundary-layer profiles, but relatively weak between the latter jets. The results are also closer to the experimental data of Lau et al. (1979) and Fleury et al. (2008) for jetBL. The peak turbulence intensities are reached at $z \simeq 17 r_{0}$ for jetBL but later at $z \simeq 22 r_{0}$ for the two other jets, which corresponds, relative to the end of the potential core, to $z \simeq z_{c}+5 r_{0}$ and $z_{c}+7 r_{0}$ respectively. They are equal to $14.3 \%$ for jetBL, but decrease approximately down to $13 \%$ for the jets with non-laminar boundarylayer profiles, see also in table 8 for the radial turbulence intensities. This trend is similar to that obtained in the mixing layers down to $z=15 r_{0}$ in figure 11.

The spectra of the centerline axial velocity fluctuations at $z=z_{c}+5 r_{0}$, i.e. roughly at the positions of the peak rms levels, are depicted in figure 22(b) as a function of $\mathrm{St}_{D}$. The spectra are superimposed and follow a $-5 / 3$ power law at $\mathrm{St}_{D} \geq 0.5$, but 
(a)

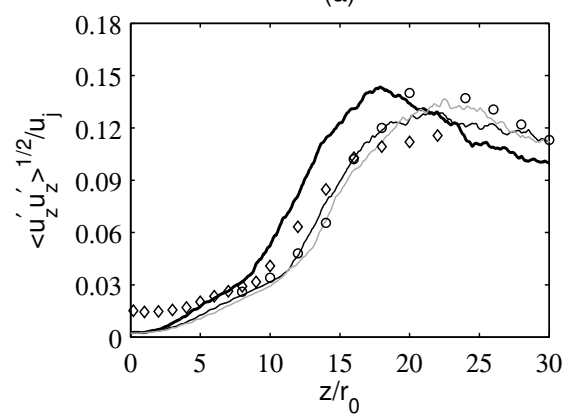

(b)

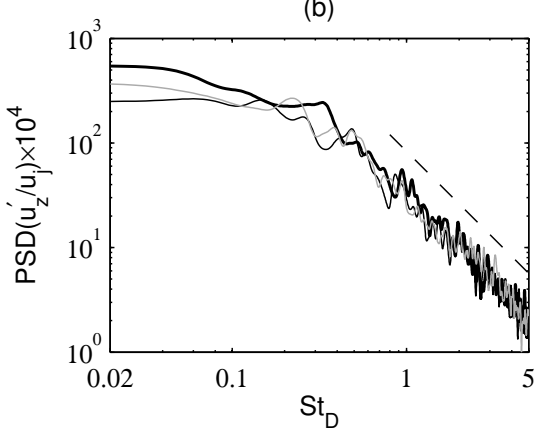

Figure 22. Properties of the centerline axial velocity fluctuations $u_{z}^{\prime}$ : (a) axial variations of rms values and (b) power spectral densities at $z=z_{c}+5 r_{0}$ as a function of $\mathrm{St}_{D}$ for - jetBL, jetT1, jetT2; same symbol types as in figure $21 ;---\mathrm{St}_{D}^{-5 / 3}$.

they significantly differ and show highest levels for jetBL at lower Strouhal numbers. Therefore, stronger large-scale structures are found not only in the mixing layers, but also downstream of the potential core for the jet with a laminar boundary-layer profile. This may be the cause for the divergence in velocity decay of figure 21(b).

The changes observed between the present jets with laminar and transitional exit mean velocity profiles are comparable to those obtained experimentally between untripped and tripped jets (Raman et al. 1989, 1994; Russ \& Strykowski 1993), as well as to those happening when the initial fluctuation level increases (Bogey et al. 2012b). In particular, in Raman et al. (1989), tripped and untripped jets at $\mathrm{M}=0.3$ and $\operatorname{Re}_{D}=6 \times 10^{5}$ with nozzle-exit turbulence intensities $u_{e}^{\prime} / u_{j} \simeq 7 \%$ and boundary-layer shape factors $\mathrm{H} \simeq 1.55$ and 1.80 , respectively, were considered. The flow development in the tripped jets is shifted by $2 r_{0}$ in the downstream direction with respect to the untripped jet, which is in line with the results of this study. However, the peak turbulence intensities on the centerline, located at $z \simeq z_{c}+7 r_{0}$, are similar in the tripped and untripped jets, which disagrees with figure 22 . The reason for this may be that the exit boundary layer of the untripped jet of Raman et al. (1989) is not laminar but transitional. This may also be due to the larger boundary-layer thickness in the simulations (Bogey \& Marsden 2013).

\subsection{Acoustic fields}

\subsubsection{Pressure snapshots}

Snapshots of the pressure fields obtained in the LES are given in figure 23. In all cases, large-scale hydrodynamic fluctuations, classically attributed to the flow coherent structures (Arndt et al. 1997), dominate within and very near the jets. Farther from the axis, sound waves emerge and propagate in the acoustic field. The waves emitted in the flow direction are strong and have long wavelengths, which is typical of the downstream subsonic jet noise component (Tam et al. 2008). Those travelling in the sideline and upstream directions are weaker and have shorter wavelengths. For the three jets, the latter ones appear to be mainly generated between $z=5 r_{0}$ and $z=10 r_{0}$. Their amplitudes, however, are visibly higher for jetBL in figure 23(a) than for jetT1 and jetT2 in figures $23(\mathrm{~b}, \mathrm{c})$.

\subsubsection{Near-field and far-field pressure levels}

The properties of the jet acoustic near fields are investigated from the pressure signals recorded at $r=L_{r}=15 r_{0}$ during the LES. Those of the jet far fields are characterized 
(a)

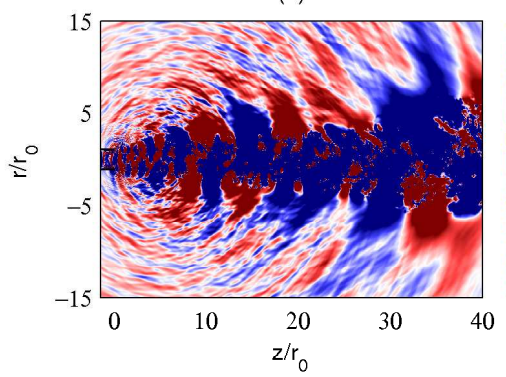

(b)

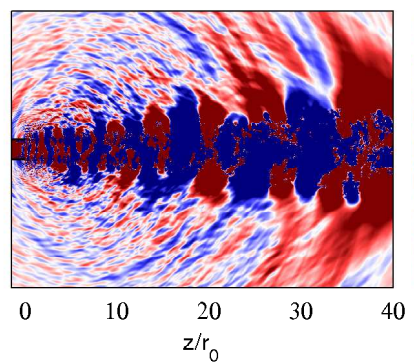

(c)

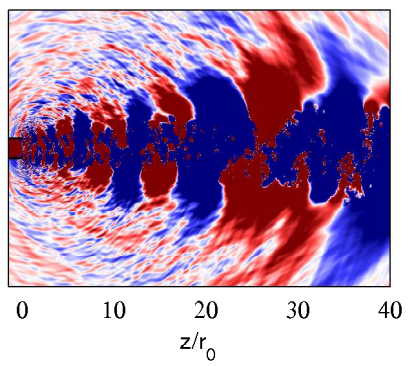

FIgure 23. (Colour available at journals . cambridge.org/flm) Snapshots in the $(z, r)$ plane of pressure fluctuations $p-p_{a}$ for (a) jetBL, (b) jetT1 and (c) jetT2. The color scale ranges from -70 to $70 \mathrm{~Pa}$, from blue to red.

from the fluctuations given at 150 radii from the nozzle exit by the two ILEE computations of sound propagation described in section 2.5. In the second case, the results presented thereafter for the angles $\phi \leq 60^{\circ}$ relative to the jet direction are obtained in the computation in which the LES data are imposed onto the ILEE grid for $r \geq 7.5 r_{0}$ at $z=L_{z}=40 r_{0}$ in order to capture most of the downstream noise components. Those for $\phi \geq 60^{\circ}$ come from the computation in which the LES/ILEE coupling at $z=L_{z}$ is carried out only for $r \geq 14 r_{0}$ to avoid the generation of significant spurious waves for large radiation angles where the noise levels are weak. It should be noted that the two far-field extrapolations provide nearly identical results at $\phi=60^{\circ}$ for Strouhal numbers greater than $\mathrm{St}_{D}=0.075$, demonstrating the negligible influence of the downstream extrapolation surface on the frequencies of interest. The overall sound pressure levels in this paper are all calculated by integrating the sound spectra from the Strouhal number value given above.

The noise levels obtained at $r=15 r_{0}$ between $z=0$ and $40 r_{0}$, and at 150 radii from the nozzle exit between $\phi=15^{\circ}$ and $150^{\circ}$ are represented in figure 24. For illustration purposes, the experimental data of Bogey et al. (2007) and Bridges \& Brown (2005) for isothermal jets at $\mathrm{M}=0.9$ and $\operatorname{Re}_{D} \simeq 10^{6}$ are also plotted. With respect to the simulated jets, these jets have 15-20 times higher Reynolds numbers and certainly quite different nozzle-exit conditions, including much thinner exit boundary layers, which may be the cause for the extra noise radiated by the jet of Bogey et al. (2007) in figure 24(a). Despite this, however, a good qualitative agreement is found with the simulation results. More importantly, for all near-field and far-field observation points, the noise levels are 2-3 dB higher for jetBL with a laminar boundary-layer profile than for the two jets with transitional profiles. In addition, the levels for jetT2 are just very slightly lower than those for jetT1. These trends are very similar to those reported for the rms values of velocity fluctuations in the jets, as expected due to the links existing between acoustic sources and turbulence intensities in subsonic jets (Zaman 1986).

The sound pressure levels obtained at $r=15 r_{0}$ for the modes $n_{\theta}=0,1$ and 2 are shown in figure 25. The levels for $n_{\theta}=0$ are maximum at $z=L_{z}=40 r_{0}$ and sharply decrease in the upstream direction, whereas those for $n_{\theta}=1$ and 2 reach a peak at $z \simeq 25 r_{0}$ and $z \simeq 20 r_{0}$, respectively. These peak positions are consistent with the the far-field directivities found experimentally for the first azimuthal modes. For instance, for the jet at $\mathrm{M}=0.6$ of Cavalieri et al. (2012), noise is strongest in the downstream direction for the axisymmetric mode and for the angles of $\phi=30^{\circ}$ for $n_{\theta}=1$ and of $\phi=40^{\circ}$ for $n_{\theta}=2$. Here, for each mode considered, the noise levels are $2-3 \mathrm{~dB}$ higher for jetBL than for jetT1 and jetT2, and the levels for the last two jets do not differ appreciably, 
(a)

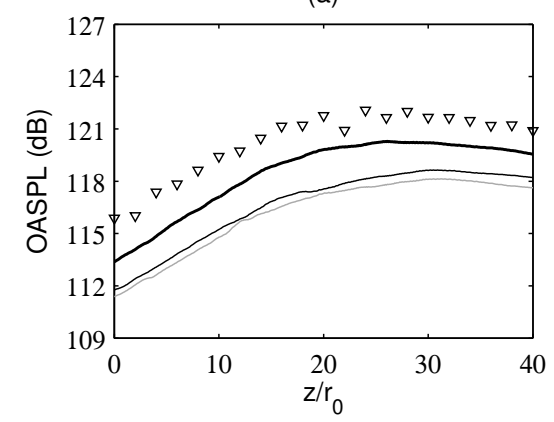

(b)

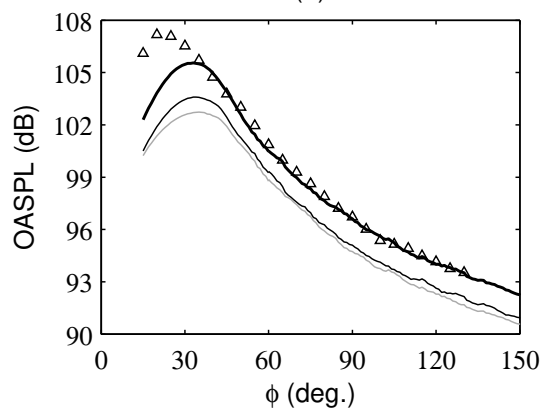

Figure 24. Overall sound pressure levels (OASPL) obtained (a) at $r=15 r_{0}$ and (b) at a distance of $150 r_{0}$ from the nozzle exit as a function of the angle $\phi$ relative to the jet direction: - jetBL, — jetT1, — jetT2; measurements for isothermal jets at $\mathrm{M}=0.9$ : $\nabla$ Bogey et al. $(2007)$ at $\operatorname{Re}_{D}=7.9 \times 10^{5}$ and $\triangle$ Bridges \& Brown $(2005)$ at $\operatorname{Re}_{D}=10^{6}$.

(a)

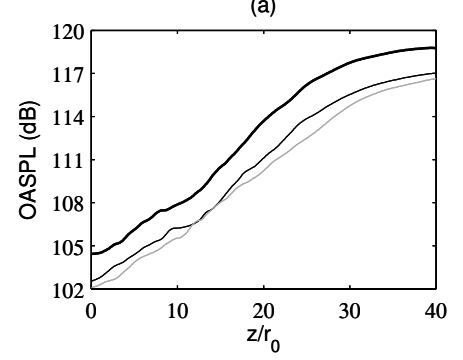

(b)

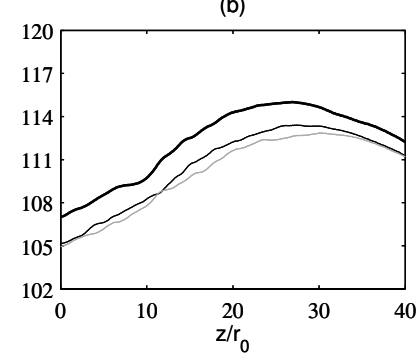

(c)

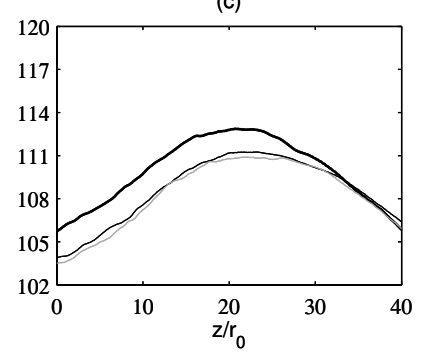

Figure 25. Overall sound pressure levels obtained at $r=15 r_{0}$ for modes (a) $n_{\theta}=0$, (b) $n_{\theta}=1$ and (c) $n_{\theta}=2:-$ jetBL, $\longrightarrow$ jetT1, $\longrightarrow$ jetT2.

just as in figure 24 for the full pressure signals. This is in line with the resemblances of the features of the full velocity flow fields and of their first modal components in the azimuthal direction, depicted in figures 17 and 19.

The pressure spectra calculated at $r=15 r_{0}$ at $z=0,20 r_{0}$ and $40 r_{0}$ are represented in figure 26 as a function of the Strouhal number $\mathrm{St}_{D}$. Those evaluated in far field for the angles of $\phi=30^{\circ}, 90^{\circ}$ and $150^{\circ}$ are provided in figure 27 . When possible, the corresponding measurements of Bogey et al. (2007) and Bridges \& Brown (2005) for jets at $\operatorname{Re}_{D} \simeq 10^{6}$ are shown. As for the overall sound levels, they compare well with the simulation results, with a better fit for the data of Bridges \& Brown (2005). The spectra for the present jets have similar shapes, typical of subsonic jet noise (Mollo-Christensen et al. 1964; Tam 1998). For small radiation angles, in figure 26(c) and figure 27(a), they are dominated by a narrow-band component centered around $\mathrm{St}_{D}=0.2$. The noise levels are 2-3 dB higher for jetBL than for the two other jets for $\mathrm{St}_{D} \leq 0.3$, but are rather close to each other for $\mathrm{St}_{D} \geq 0.6$. This can be related to the velocity spectra of figures 17 (f) and 22(b) obtained near the end of the potential core, where the downstream acoustic components originate (Panda et al. 2005; Bogey \& Bailly 2007; Tam et al. 2008; Bogey 2019), which also contain stronger low-frequency components for jetBL but are superimposed at high frequencies. For large radiation angles, in figures 26(a,b) and $27(\mathrm{~b}, \mathrm{c})$, the pressure spectra are broadband. In that case, the emitted sound is louder for jetBL than for jetT1 and jetT2 not only at $\mathrm{St}_{D} \leq 0.3$ as previously, but also at higher Strouhal numbers. In particular, an increase of $1-1.5 \mathrm{~dB}$ is noted over $1.2 \leq \mathrm{St}_{D} \leq 4.8$. This most likely results from the higher turbulence intensities in the mixing layers for 
(a)

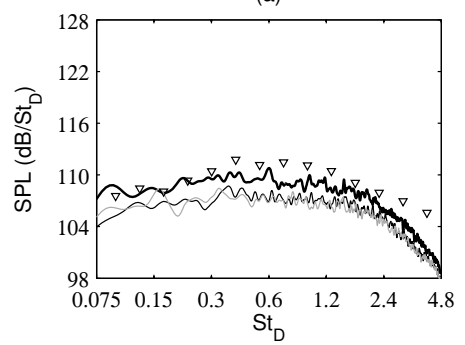

(b)

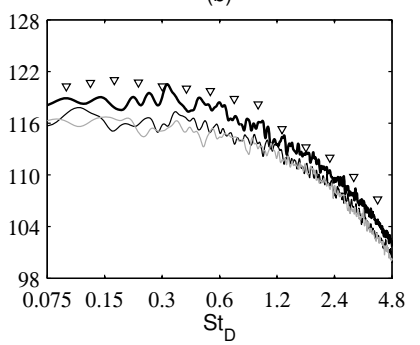

(c)

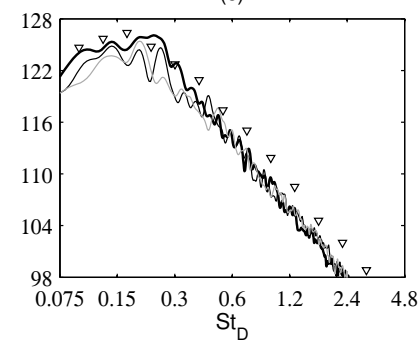

Figure 26. Sound pressure levels (SPL) obtained at $r=15 r_{0}$ at (a) $z=0$, (b) $z=20 r_{0}$ and (c) $z=40 r_{0}$, as a function of $\mathrm{St}_{D}$ : $\longrightarrow$ jetBL, $\longrightarrow$ jetT1, $\longrightarrow$ jetT2; $\nabla$ measurements of Bogey et al. (2007) for an isothermal jet at $\mathrm{M}=0.9$ and $\operatorname{Re}_{D}=7.9 \times 10^{5}$.

jetBL, in a region where the acoustic sources have a wide range of frequencies (Chu \& Kaplan 1976; Fisher et al. 1977; Narayanan et al. 2002; Lee \& Bridges 2005). The difference at $\mathrm{St}_{D} \geq 3.2$ is however rather surprising given the velocity spectra of figures 17 and 22, none of which exhibits stronger components at such high Strouhal numbers for jetBL.

It is difficult to compare the present results with the experimental data available for tripped and untripped jets, because tripping usually mainly results in removing the noise generated by the vortex pairings occurring in fully laminar jets (Zaman 1985a; Bridges \& Hussain 1987; Bogey \& Bailly 2010; Bogey et al. 2012b). Nevertheless, they bear significant similarities with the results obtained for the jets exhausting from the ASME and the conical nozzles (Viswanathan \& Clark 2004; Zaman 2012; Karon \& Ahuja 2013). Indeed, approximately $2 \mathrm{~dB}$ more noise is emitted in the first case, which was attributed by Zaman (2012) to the fact that the exit boundary layers are nominally laminar with the ASME nozzle, but turbulent with the conical nozzle. This hypothesis was further supported by Karon \& Ahuja (2013) who measured lower boundary-layer shape factors for the conical nozzle and found, for instance, $\mathrm{H}=2.34$ in the ASME case but $\mathrm{H}=1.71$ in the conical case for $\mathrm{M}=0.4$, as indicated in table 1 . The difference in noise level between the ASME and the conical nozzles is maximum at frequencies typically one decade higher than the jet noise peak frequencies, and is stronger for $\phi=90^{\circ}$ than for $\phi=30^{\circ}$. Neither of these trends are observed in this work. This may be due to the thick boundary layers in the simulations, yielding a peak Strouhal number of only $\mathrm{St}_{D}=1.20$ early on in the shear layers of jetBL. By making the boundary-layer/shear-layer transition happen over a distance of $5 r_{0}-6 r_{0}$ for jetBL, the thick exit velocity profiles also allow the effects of the boundary-layer shape on the mixing-layer turbulent structures to persist, as pointed out in section 3.3.2, down to the end of the potential core, where low-frequency sound waves are radiated in the downstream direction. Thus, it can be assumed that with a thinner boundary layer, the extra noise components for the jet with a laminar nozzle-exit mean velocity profile would emerge at higher frequencies, and would be lower for small emission angles, leading to a better agreement with the ASME case.

\section{Conclusion}

The influence of the nozzle-exit velocity profile has been investigated for isothermal round jets at a Mach number of $\mathrm{M}=0.9$ and a Reynolds number of $\operatorname{Re}_{D}=5 \times 10^{4}$ with boundary-layer momentum thicknesses of $2.8 \%$ of the jet radius and peak turbulence intensities of $6 \%$ at the exit of pipe nozzle. One jet with a laminar boundary-layer profile of shape factor $\mathrm{H}=2.29$ and two jets with transitional profiles with $\mathrm{H}=1.71$ 

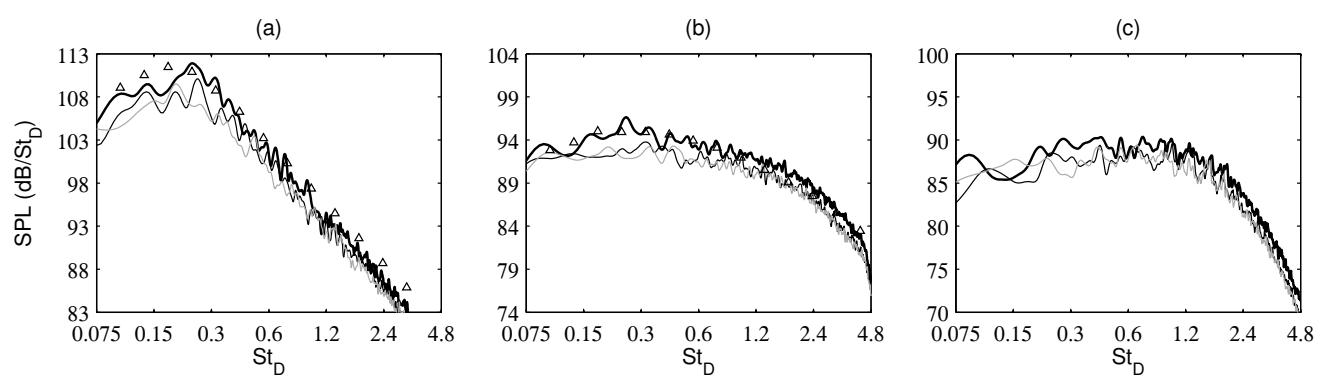

FIGURE 27. Sound pressure levels obtained at $150 r_{0}$ from the nozzle exit for (a) $\phi=30^{\circ}$, (b) $\phi=90^{\circ}$ and (c) $\phi=150^{\circ}$, as a function of $\mathrm{St}_{D}:-$ jetBL, - jetT1, jetT2; $\triangle$ measurements of Bridges \& Brown (2005) for an isothermal jet at $\mathrm{M}=0.9$ and $\operatorname{Re}_{D}=10^{6}$.

and 1.96 are considered. The jet flow and sound fields computed for the laminar profile differ significantly from those for the two transitional profiles. The latter ones are very close to each other, suggesting that similar results would be obtained for a turbulent profile. In the non-laminar cases, the jets develop more slowly, the turbulence intensities are lower in the mixing layers but also just downstream of the jet potential core, and less noise is emitted in the acoustic field. Due to the sharper velocity gradient very near the nozzle, the initial shear-layer instability waves also grow more rapidly and at higher frequencies, in agreement with the predictions of a linear stability analysis performed from the simulation profiles. Compared to the peak unstable frequencies in a mixing layer of same momentum thickness, these frequencies are similar for the jet with a laminar boundary-layer profile, but greater for the two other ones. As a result, the initial instability waves persist over a larger distance in the laminar case, organizing the flow and leading to stronger large-scale structures downstream of the boundary-layer/mixing-layer transition, than in the non-laminar cases.

By combining high-fidelity computations of jets with well-controlled upstream conditions and linear stability analyses, this study suggests explanations for and connections between some flow and acoustic features of free shear flows and jets, which have observed experimentally for years or even decades but whose reasons are still unclear. This is the case for the discrepancy in frequency of the initial instability waves between initially laminar and initially turbulent conditions. The present results show that this discrepancy is due to the fact that the most unstable frequencies near the nozzle are fixed by the maximum velocity gradient and not by the boundary-layer momentum thickness. Concerning the controversial issue of the persistence of coherent structures in turbulent mixing layers, it is found that that such structures are more likely to form for a laminar boundary-layer profile than for a non-laminar profile, because of the continuity of the peak instability-wave frequencies during the changeover from a boundary-layer to a mixing-layer profile in the first case, but of their significant decrease in the other one. Thus, it becomes easier to understand why for some nozzles such as the ASME nozzle, at the exit of which the flow is highly disturbed but the mean velocity profile is laminar, intense large-scale structures appear in the mixing layers and additional noise is measured in the acoustic field.

In this paper, in order to ensure a high numerical accuracy at a reasonable computational cost, the effects of the boundary-layer velocity profile have been investigated for a jet at a Reynolds number only of $\mathrm{Re}_{D}=5 \times 10^{4}$ with thick boundary layers. Of course, it would be interesting to consider jets at higher Reynolds numbers with thinner boundary layers in further simulations to get closer to the conditions encountered in the 
(a)

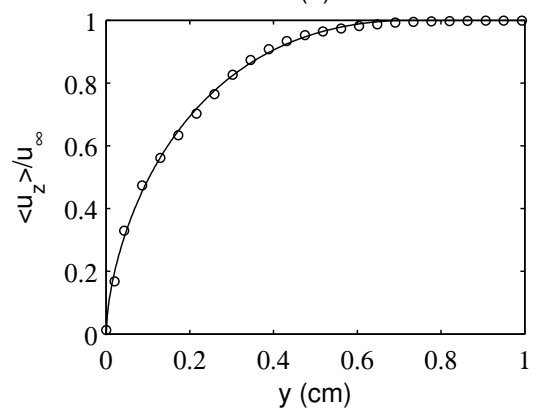

(b)

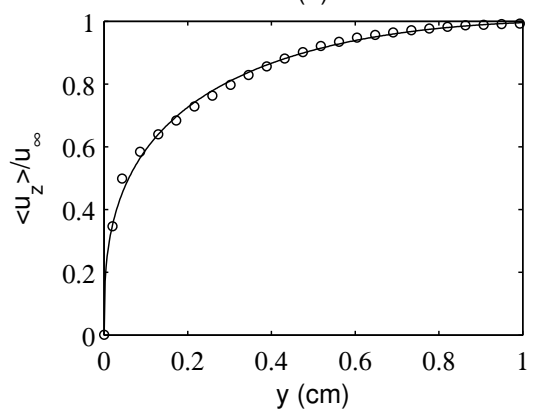

FIGURE 28. Representation of boundary-layer mean velocity profiles measured by Schubauer \& Klebanoff (1955) close to the laminar-turbulent transition and of profiles given by equation (2.2) with $y=r_{0}-r$ : (a) $\circ$ measurements at $x=1.91 \mathrm{~m}$ and - profile T1 with $\delta_{T_{1}}=0.73 \mathrm{~cm}$, (b) $\circ$ measurements at $x=2.06 \mathrm{~m}$ and $\longrightarrow$ profile $\mathrm{T} 2$ with $\delta_{T_{2}}=1.17 \mathrm{~cm}$.

laboratory-scale experiments of the literature. New experiments detailing the shear-layer turbulence properties just downstream of the nozzle for laminar and turbulent nozzle-exit velocity profiles would also be a useful complement of the present work.

\section{Acknowledgments}

This work was granted access to the HPC resources of FLMSN (Fédération Lyonnaise de Modélisation et Sciences Numériques), partner of EQUIPEX EQUIP@MESO, and of the resources of CINES (Centre Informatique National de l'Enseignement Supérieur) and IDRIS (Institut du Développement et des Ressources en Informatique Scientifique) under the allocation 2018-2a0204 made by GENCI (Grand Equipement National de Calcul Intensif). It was performed within the framework of the Labex CeLyA of Université de Lyon, within the programme 'Investissements d'Avenir' (ANR-10-LABX-0060/ANR-16IDEX-0005) operated by the French National Research Agency (ANR).

\section{Appendix A}

In the simulations of jetT1 and jetT2, the axial velocity profiles $\mathrm{T} 1$ and $\mathrm{T} 2$ given by equation (2.2) with $i=1$ and 2 are imposed at the pipe-nozzle inlet at $z=-2 r_{0}$. Considering the strong similarities between the near-wall mean-flow statistics obtained for turbulent pipe and boundary layer flows (Monty et al. 2009), they have been designed to fit the experimental data provided by Schubauer \& Klebanoff (1955) for a boundary layer over a flat plate in the region of laminar-turbulent flow transition at two axial positions. For the comparison, the measured profiles and the $\mathrm{T} 1$ and $\mathrm{T} 2$ profiles are represented in figure 28 as a function of the distance to the wall as in the experiment, using the boundary-layer thicknesses of $\delta_{T_{1}}=0.73 \mathrm{~cm}$ and $\delta_{T_{2}}=1.17 \mathrm{~cm}$ in equation $(2.2)$. In both cases, a very good agreement is observed close to the wall as well as far away from it.

\section{Appendix B}

In a preliminary grid-sensitivity study, simulations of jetT1 and jetT2 have been performed using two grids extending in the axial direction, excluding the outflow sponge zones, only down to $z=4 r_{0}$ in order to save computational time. The coarsest of the 
(a)

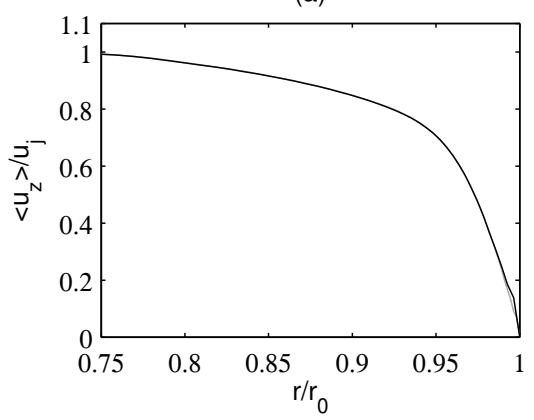

(b)

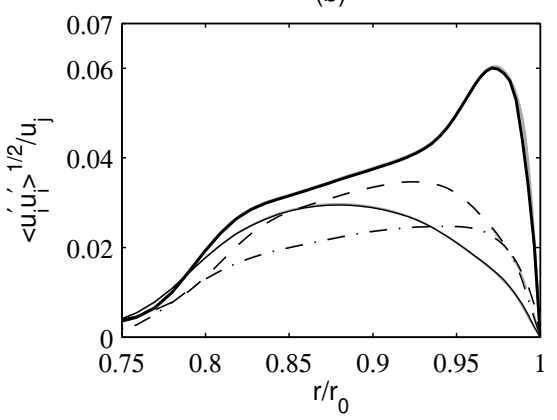

Figure 29. Nozzle-exit profiles (a) of mean axial velocity $\left\langle u_{z}\right\rangle$ and (b) of turbulence intensities $-\left\langle u_{z}^{\prime 2}\right\rangle^{1 / 2} / u_{j},-\left\langle u_{r}^{\prime 2}\right\rangle^{1 / 2} / u_{j},---\left\langle u_{\theta}^{\prime 2}\right\rangle^{1 / 2} / u_{j}$ and $-\cdot-\cdot\left\langle u_{r}^{\prime} u_{z}^{\prime}\right\rangle^{1 / 2} / u_{j}$ obtained for jetT2 using (black) $\Delta r / r_{0}=0.36 \%$ and (grey) $\Delta r / r_{0}=0.18 \%$ at $r=r_{0}$.

(a)

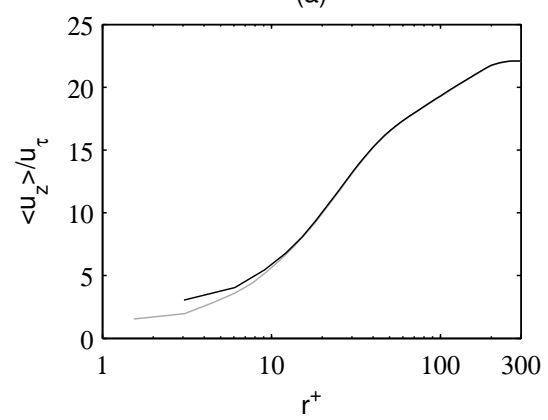

(b)

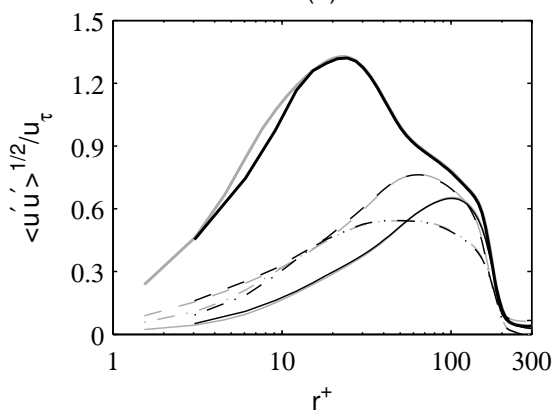

Figure 30. Nozzle-exit profiles (a) of mean axial velocity and (b) of turbulence intensities, represented in wall units based on the wall friction velocity using the same linetypes as in figure 29 .

two grids coincides with the grid used for the full jet LES, defined in table 3, in the boundary-layer region. The finest grid is identical to the coarsest one in the directions $\theta$ and $z$, but differs in the radial direction with $\Delta r / r_{0}=0.18 \%$ instead of $\Delta r / r_{0}=0.36 \%$ at $r=r_{0}$. In the two additional LES, the tripping procedure is exactly the same as in the jet LES. In the LES using the finest grid, however, the time step is twice as small because of the numerical stability condition, leading to an application of the relaxation filtering that is twice as frequent. The flow properties obtained using the two different grids at the nozzle exit are found to be nearly identical. Consequently, they depend neither on the wall-normal spacing, nor on the explicit filtering applied to remove grid-to-grid oscillations as well as to relax subgrid-scale turbulent energy.

By way of illustration, the nozzle-exit profiles of mean axial velocity and of turbulence intensities obtained for jetT2, that is for the jet with the sharpest boundary-layer profile, are represented in figure 29 using outer units and in figure 30 using wall units. The solutions calculated using the two grids with $\Delta r / r_{0}=0.36 \%$ and $\Delta r / r_{0}=0.18 \%$ at the wall superpose or are very close to each other.

\section{REFERENCES}

Ahuja, K. K., Tester, B. J. \& Tanna, H. K. 1987 Calculation of far field jet noise spectra from near field measurements with true source location. J. Sound Vib. 116 (3), 415-426. 
Arndt, R. E. A., Long, D. F. \& Glauser, M. N. 1997 The proper orthogonal decomposition of pressure fluctuations surrounding a turbulent jet. J. Fluid Mech. 340, 1-33.

Berland, J., Bogey, C., Marsden, O. \& Bailly, C. 2007 High-order, low dispersive and low dissipative explicit schemes for multi-scale and boundary problems. J. Comput. Phys. 224 (2), 637-662.

Bogey, C. 2018 Grid sensitivity of flow field and noise of high-reynolds-number jets computed by large-eddy simulation. Int. J. Aeroacoust. 17 (4-5), 399-424.

Bogey, C. 2019 On noise generation in low reynolds number temporal round jets at a mach number of 0.9. J. Fluid Mech. 859, 1022-1056.

Bogey, C. \& BAilly, C. 2002 Three-dimensional non reflective boundary conditions for acoustic simulations: far-field formulation and validation test cases. Acta Acust. united Ac. 88 (4), 463-471.

Bogey, C. \& Bailly, C. 2004 A family of low dispersive and low dissipative explicit schemes for flow and noise computations. J. Comput. Phys. 194 (1), 194-214.

Bogey, C. \& BAilly, C. 2006 Large eddy simulations of transitional round jets: influence of the reynolds number on flow development and energy dissipation. Phys. Fluids 18 (6), 065101.

Bogey, C. \& BAILly, C. 2007 An analysis of the correlations between the turbulent flow and the sound pressure field of subsonic jets. J. Fluid Mech. 583, 71-97.

Bogey, C. \& BAilly, C. 2009 Turbulence and energy budget in a self-preserving round jet: direct evaluation using large-eddy simulation. J. Fluid Mech. 627, 129-160.

Bogey, C. \& BAilly, C. 2010 Influence of nozzle-exit boundary-layer conditions on the flow and acoustic fields of initially laminar jets. J. Fluid Mech. 663, 507-539.

Bogey, C., Barré, S. \& BAilly, C. 2008 Direct computation of the noise generated by subsonic jets originating from a straight pipe nozzle. Int. J. Aeroacoust. 7 (1), 1-22.

Bogey, C., Barré, S., Fleury, V., Bailly, C. \& Juvé, D. 2007 Experimental study of the spectral properties of near-field and far-field jet noise. Int. J. Aeroacoust. 6 (2), 73-92.

Bogey, C., Barré, S., Juvé, D. \& BAilly, C. 2009 a Simulation of a hot coaxial jet: direct noise prediction and flow-acoustics correlations. Phys. Fluids 21 (3), 035105.

Bogey, C., De Cacqueray, N. \& Bailly, C. $2009 b$ A shock-capturing methodology based on adaptative spatial filtering for high-order non-linear computations. J. Comput. Phys. 228 (5), 1447-1465.

Bogey, C., De Cacqueray, N. \& Bailly, C. $2011 a$ Finite differences for coarse azimuthal discretization and for reduction of effective resolution near origin of cylindrical flow equations. J. Comput. Phys. 230 (4), 1134-1146.

Bogey, C. \& MARsden, O. 2013 Identification of the effects of the nozzle-exit boundary-layer thickness and its corresponding reynolds number in initially highly disturbed subsonic jets. Phys. Fluids 25 (5), 055106.

Bogey, C. \& Marsden, O. 2016 Simulations of initially highly disturbed jets with experimentlike exit boundary layers. AIAA J. 54 (4), 1299-1312.

Bogey, C., Marsden, O. \& Bailly, C. $2011 b$ Large-eddy simulation of the flow and acoustic fields of a reynolds number $10^{5}$ subsonic jet with tripped exit boundary layers. Phys. Fluids 23 (3), 035104.

Bogey, C., Marsden, O. \& Bailly, C. 2011c On the spectra of nozzle-exit velocity disturbances in initially nominally turbulent jets. Phys. Fluids 23 (9), 091702.

Bogey, C., Marsden, O. \& Bailly, C. $2012 a$ Effects of moderate reynolds numbers on subsonic round jets with highly disturbed nozzle-exit boundary layers. Phys. Fluids 24 (10), 105107.

Bogey, C., Marsden, O. \& Bailly, C. $2012 b$ Influence of initial turbulence level on the flow and sound fields of a subsonic jet at a diameter-based reynolds number of $10^{5}$. J. Fluid Mech. 701, 352-385.

Bradshaw, P. 1966 The effect of initial conditions on the development of a free shear layer. $J$. Fluid Mech. 26 (2), 225-236.

Brès, G. A., Jordan, P., Jaunet, V., Le Rallic, M., Cavalieri, A. V. G., Towne, A., Lele, S. K., Colonius, T. \& Schmidt, O. T. 2018 Importance of the nozzle-exit boundary-layer state in subsonic turbulent jets. J. Fluid Mech. 851, 83-124.

Bridges, J. \& Brown, C. A. 2005 Validation of the small hot jet acoustic rig for aeroacoustics. Tech. Rep. 2005-2846. AIAA Paper. 
Bridges, J. E. \& Hussain, A. K. M. F. 1987 Roles of initial conditions and vortex pairing in jet noise. J. Sound Vib. 117 (2), 289-311.

Browand, F. K. \& Latigo, B. O. 1979 Growth of the two-dimensional mixing layer from a turbulent and nonturbulent boundary layer. Phys. Fluids 22 (6), 1011-1019.

Brown, G. L. \& Roshko, A. 1974 On density effects and large structure in turbulent mixing layers. J. Fluid Mech. 64 (4), 775-816.

Bühler, S., Kleiser, L. \& Bogey, C. 2014 Simulation of subsonic turbulent nozzle-jet flow and its near-field sound. AIAA J. 52 (8), 1653-1669.

Castelain, T. 2006 Contrôle de jet par microjets impactants. Mesure de bruit rayonné et analyse aérodynamique. PhD thesis, Ecole Centrale de Lyon, France, no. 2006-33.

Castillo, L. \& Johansson, T. G. 2012 The effects of the upstream conditions on a low reynolds number turbulent boundary layer with zero pressure gradient. J. Turbul. 3, 031.

Cavalieri, A. V. G., Jordan, P., Colonius, T. \& Gervais, Y. 2012 Axisymmetric superdirectivity in subsonic jets. J. Fluid Mech. 704, 388-420.

Chandrsuda, C., Mehta, R. D., Weir, A. D. \& Bradshaw, P. 1978 Effect of free-stream turbulence on large structure in turbulent mixing layers. J. Fluid Mech. 85 (4), 693-704.

Chu, W. T. \& KAPlan, R. E. 1976 Use of a spherical concave reflector for jet-noise-source distribution diagnosis. J. Acoust. Soc. Am. 59 (6), 1268-1277.

Coles, D. E. 1962 The turbulent boundary layer in a compressible fluid. Tech. Rep. R-403-PR. Rand Corp.

Crighton, D. G. 1981 Acoustics as a branch of fluid mechanics. J. Fluid Mech. 106, 261-298.

Crow, S. C. \& Champagne, F. H. 1971 Orderly structure in jet turbulence. J. Fluid Mech. 48, 547-591.

De Chant, L. J. 2005 The venerable 1/7th power law turbulent velocity profile: a classical nonlinear boundary value problem solution and its relationship to stochastic processes. Appl. Math. Comput. 161 (2), 463-474.

Drubka, R. E. \& Nagib, H. M. 1981 Instabilities in near field of turbulent jets and their dependence on initial conditions and reynolds number. Tech. Rep. R-81-2. IIT Fluids \& Heat Transfer Report.

Erm, P. L. \& Joubert, P. N. 1991 Low-reynolds-number turbulent boundary layers. J. Fluid Mech. 230, 1-44.

Fauconnier, D., Bogey, C. \& Dick, E. 2013 On the performance of relaxation filtering for large-eddy simulation. J. Turbulence 14 (1), 22-49.

Fernholz, H. H. \& Finley, P. J. 1996 The incompressible zero-pressure-gradient turbulent boundary layer: an assessment of the data. Prog. Aerosp. Sci. 32 (4), 245-311.

Fieldler, H. E. 1988 Coherent structures in turbulent flows. Prog. Aerosp. Sci. 25, 231-269.

Fisher, M. J., Harper-Bourne, M. \& GlegG, S. A. L. 1977 Jet engine noise source location: The polar correlation technique. J. Sound Vib. 51 (1), 23-54.

Fleury, V. 2006 Superdirectivité, bruit d'appariement et autres contributions au bruit de jet subsonique. PhD thesis, Ecole Centrale de Lyon, France, no. 2006-18.

Fleury, V., Bailly, C., Jondeau, E., Michard, M. \& Juvé, D. 2008 Space-time correlations in two subsonic jets using dual-piv measurements. AIAA J. 46 (10), 2498-2509.

Fontaine, R. A., Elliott, G. S., Austin, J. M. \& Freund, J. B. 2015 Very near-nozzle shear-layer turbulence and jet noise. J. Fluid Mech. 770, 27-51.

Gloerfelt, X. \& Berland, J. 2012 Turbulent boundary layer noise: direct radiation at mach number 0.5. J. Fluid Mech. 723, 318-351.

Gutmark, E. \& Ho, C.-M. 1983 Preferred modes and the spreading rates of jets. Phys. Fluids 26 (10), 2932-2938.

Harper-Bourne, M. 2010 Jet noise measurements: past and present. Int. J. Aeroacoust. 9 (4 \& 5), 559-588.

Hill, W. G., Jenkins, R. C. \& Gilbert, B. L. 1976 Effects of the initial boundary-layer state on turbulent jet mixing. AIAA J. 14 (11), 1513-1514.

Ho, C. \& Huerre, P. 1984 Perturbed free shear layers. Annu. Rev. Fluid Mech. 16, 365-422.

Husain, Z. D. \& Hussain, A. K. M. F. 1979 Axisymmetric mixing layer: influence of the initial and boundary conditions. AIAA J. 17 (1), 48-55.

Hussain, A. K. M. F. 1986 Coherent structures and turbulence. J. Fluid Mech. 173, 303-356. 
Hussain, A. K. M. F. \& Zaman, K. B. M. Q. 1985 An experimental study of organized motions in the turbulent plane mixing layer. J. Fluid Mech. 159, 85-104.

Hussain, A. K. M. F. \& Zedan, M. F. 1978 a Effects of the initial condition on the axisymmetric free shear layer: Effects of the initial fluctuation level. Phys. Fluids 21 (9), 1475-1481.

Hussain, A. K. M. F \& Zedan, M. F. $1978 b$ Effects of the initial condition on the axisymmetric free shear layer: Effects of the initial momentum thickness. Phys. Fluids 21 (7), 1100-1112.

Hutchings, N. 2012 Caution: tripping hazards. J. Fluid Mech. 710, 1-4.

Karon, A. Z. \& Ahuja, K. K. 2013 Effect of nozzle-exit boundary layer on jet noise. Tech. Rep. 2013-0615. AIAA Paper.

Kim, J., Moin, P., \& Moser, R. 1987 Turbulence statistics in fully developed channel flow at low reynolds number. J. Fluid Mech. 177, 133-166.

Klebanoff, P. S. \& Diehl, Z. W. 1952 Some features of artificially thickened fully developed turbulent boundary layers with zero pressure gradient. Tech. Rep. 1110. NACA TN.

Kremer, F. \& Bogey, C. 2015 Large-eddy simulation of turbulent channel flow using relaxation filtering: resolution requirement and reynolds number effects. Comput. Fluids 116, 17-28.

LAU, J. C., Morris, P. J. \& Fisher, M. J. 1979 Measurements in subsonic and supersonic free jets using a laser velocimeter. J. Fluid Mech. 93 (1), 1-27.

Lee, S. S. \& Bridges, J. 2005 Phased-array measurements of single flow hot jets. Tech. Rep. 2005-213826. NACA TM.

Lilley, G. M. 1994 Jet noise classical theory and experiments. In Aeroacoustics of Flight Vehicles (ed. H. H. Hubbard), , vol. 1, pp. 211-289. Acoustical Society of America.

Lorteau, M., Cléro, F. \& Vuillot, F. 2015 Analysis of noise radiation mechanisms in hot subsonic jet from a validated large eddy simulation solution. Phys. Fluids 27 (7), 075108.

Maestrello, L. \& MCDAid, E. 1971 Acoustic characteristics of a high-subsonic jet. AIAA J. 9 (6), 1058-1066.

Michalke, A. 1984 Survey on jet instability theory. Prog. Aerosp. Sci. 21, 159-199.

Mohseni, K. \& Colonius, T. 2000 Numerical treatment of polar coordinate singularities. J. Comput. Phys. 157 (2), 787-795.

Mollo-Christensen, E., Kolpin, M. A. \& Martucelli, J. R. 1964 Experiments on jet flows and jet noise far-field spectra and directivity patterns. J. Fluid Mech. 18 (2), 285-301.

Monty, J. P., Hutchins, N., NG, H. C. H., Marusic, I. \& Chong, M. S. 2009 A comparison of turbulent pipe, channel and boundary layer flows. J. Fluid Mech. 632, 431- 442 .

Morris, P. J. 1976 The spatial viscous instability of axisymmetric jets. J. Fluid Mech. 77 (3), $511-529$.

Morris, P. J. 2010 The instability of high speed jets. Int. J. Aeroacoust. 9 (1-2), 1-50.

Morris, P. J. \& Zaman, K. B. M. Q. 2009 Velocity measurements in jets with application to noise source modelling. J. Sound Vib. 329 (4), 394-414.

Morris, S. C. \& Foss, J. F. 2003 Turbulent boundary layer to single-stream shear layer: the transition region. J. Fluid Mech. 494, 187-221.

Narayanan, S., Barber, T. J. \& Polak, D. R. 2002 High subsonic jet experiments: Turbulence and noise generation studies. AIAA J. 40 (3), 430-437.

Panda, J., Seasholtz, R. G. \& Elam, K. A. 2005 Investigation of noise sources in high-speed jets via correlation measurements. J. Fluid Mech. 537, 349-385.

Raman, G., Rice, E. J. \& Reshotko, E. 1994 Mode spectra of natural disturbances in a circular jet and the effect of acoustic forcing. Exp. Fluids 17, 415-426.

Raman, G., Zaman, K. B. M. Q. \& Rice, E. J. 1989 Initial turbulence effect on jet evolution with and without tonal excitation. Phys. Fluids A 1 (7), 1240-1248.

Russ, S. \& STRYkowski, P. J. 1993 Turbulent structure and entrainment in heated jets: The effect of initial conditions. Phys. Fluids A 5 (12), 3216-3225.

Sabatini, R. \& Bailly, C. 2015 Numerical algorithm for computing acoustic and vortical spatial instability waves. AIAA J. 53 (3), 692-702.

Sandberg, R. D., Sandham, N. D. \& Suponitsky, V. 2012 DNS of compressible pipe flow exiting into a coflow. Int. J. Heat and Fluid Flow 35, 33-44.

SAto, H. 1971 Experimental investigation on the transition of laminar separated layer. J. Phys. Soc. Jpn. 48, 702-709. 
Schlatter, P. \& ÖRlü, R. 2012 Turbulent boundary layers at moderate reynolds numbers: inflow length and tripping effects. J. Fluid Mech. 710, 5-34.

Schubauer, G. B. \& Klebanoff, P. S. 1955 Contributions on the mechanics of boundarylayer transition. Tech. Rep. 3498. NACA TN.

Spalart, P. R. 1988 Direct simulation of a turbulent boundary layer up to $r_{\theta}=1410$. J. Fluid Mech. 187, 61-98.

TAM, C. K. W. 1998 Jet noise: Since 1952. Theor. Comput. Fluid Dyn. 10 (1-4), 393-405.

TAM, C. K. W \& Dong, Z. 1996 Radiation and outflow boundary conditions for direct computation of acoustic and flow disturbances in a nonuniform mean flow. J. Comput. Acous. $4(2), 175-201$.

Tam, C. K. W., Viswanathan, K., Ahuja, K. K. \& Panda, J. 2008 The sources of jet noise: experimental evidence. J. Fluid Mech. 615, 253-292.

TAnnA, H. K. 1977 An experimental study of jet noise. part i: Turbulent mixing noise. J. Sound Vib. 50 (3), 405-428.

Tomkins, C. D. \& Adrian, R. J. 2005 Energetic spanwise modes in the logarithmic layer of a turbulent boundary layer. J. Fluid Mech. 545, 141-162.

Uzun, A. \& Hussaini, M. 2007 Investigation of high frequency noise generation in the nearnozzle region of a jet using large eddy simulation. Theor. Comput. Fluid Dyn. 21 (4), 291-321.

Viswanathan, K. 2004 Aeroacoustics of hot jets. J. Fluid Mech. 516, 39-82.

ViswanATHAN, K. 2006 Distributions of noise sources in heated and cold jets: are they different? Int. J. Aeroacoust. 9 (4 \& 5), 589-626.

Viswanathan, K. \& Clark, L. T. 2004 Effect of nozzle internal contour on jet aeroacoustics. Int. J. Aeroacoust. 3 (2), 103-135.

Wygnanski, I., Oster, D., Fiedler, H. \& Dziomba, B. 1979 On the perseverance of a quasi-two-dimensional eddy-structure in a turbulent mixing layer. J. Fluid Mech. 93 (2), $325-335$.

Xu, G. \& Antonia, R. A. 2002 Effects of different initial conditions on a turbulent free jet. Exp. Fluids 33, 677-683.

Yule, A. J. 1978 Large-scale structure in the mixing layer of a round jet. J. Fluid Mech. 89 (3), $413-432$.

Zaman, K. B. M. Q. 1985 a Effect of initial condition on subsonic jet noise. AIAA J. 23 (9), $1370-1373$

Zaman, K. B. M. Q. $1985 b$ Far-field noise of a subsonic jet under controlled excitation. J. Fluid Mech. 152, 83-111.

Zaman, K. B. M. Q. 1986 Flow field and near and far sound field of a subsonic jet. J. Sound Vib. 106 (1), 1-16.

Zaman, K. B. M. Q. 2012 Effect of initial boundary-layer state on subsonic jet noise. AIAA J. 50 (8), 1784-1795.

Zaman, K. B. M. Q. 2017 Increased jet noise due to a "nominally laminar" state of nozzle exit boundary layer. Tech. Rep. 2017-219440. NASA TM.

Zaman, K. B. M. Q. \& Hussain, A. K. M. F. 1981 Turbulence suppression in free shear flows by controlled excitation. J. Fluid Mech. 103, 133-159.

Zhu, M., Pérez Arroyo, C., Fosso Pounangué, A., Sanjosé, M. \& Moreau, S. 2018 Isothermal and heated subsonic jet noise using large eddy simulations on unstructured grids. Comput. Fluids 171, 166-192. 